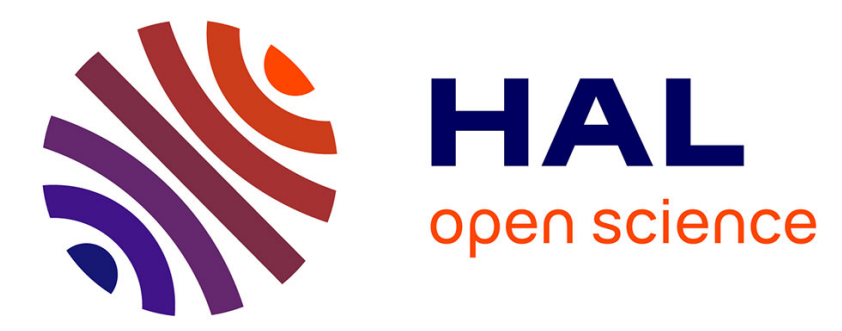

\title{
Basic statistical recipes for the emergence of biochemical discernment.
}

Denis Michel

\section{To cite this version:}

Denis Michel. Basic statistical recipes for the emergence of biochemical discernment.. Progress in Biophysics and Molecular Biology, 2011, 106 (3), pp.498-516. 10.1016/j.pbiomolbio.2011.07.011. hal-00627619

\section{HAL Id: hal-00627619 \\ https://hal.science/hal-00627619}

Submitted on 29 Sep 2011

HAL is a multi-disciplinary open access archive for the deposit and dissemination of scientific research documents, whether they are published or not. The documents may come from teaching and research institutions in France or abroad, or from public or private research centers.
L'archive ouverte pluridisciplinaire HAL, est destinée au dépôt et à la diffusion de documents scientifiques de niveau recherche, publiés ou non, émanant des établissements d'enseignement et de recherche français ou étrangers, des laboratoires publics ou privés. 


\title{
Basic statistical recipes for the emergence of biochemical discernment
}

\author{
Denis Michel \\ Université de Rennes1. Molecular and Cellular Interactions UMR6026. Irset. IFR140GFAS. \\ Bat.13 Campus de Beaulieu 35042 Rennes cedex France.denis.michel@live.fr
}

\begin{abstract}
An essential step towards understanding life would be to identify the very basic mechanisms responsible for the discerning behaviour of living biochemical systems, absent from randomly reacting chemical soups. One intuitively feels that this question goes beyond the particular nature of the biological molecules and should relate to general physical principles. The preeminent physicist Ludwig Boltzmann early envisioned life as a struggle for entropy, in concordance with the subsequent principle of self-organization out of equilibrium. Reexamination of elementary steady state biochemical systems from a statistical perspective supports this view and shows that sigmoidal responses arising from microstates elimination, are sufficient to explain innermost characteristics of life, including its capacity to convert random molecular interactions into accurate biological reactions. A primary operating strategy to achieve this goal is the introduction of time-irreversible transitions in molecular state conversion cycles by injection of free energy, which confers decisional capacity to single macromolecules. Selected examples from various fields of molecular biology such as enzymology and gene expression, are provided to show that these non-equilibrium steady state mechanisms remain important in contemporary biochemical systems. But in addition, information archiving allowed the emergence of the time-reversible counterparts of these mechanisms, mediated by evolutionary preorganized macromolecular complexes capable of increasing discernment in a non-dissipative manner.
\end{abstract}

Keywords: Entropy; cooperativity; induced fit; information; life; Brillouin. 


\section{Introduction}

The present study focuses on an original aspect of life: the biophysical principles responsible for the discernment of biochemical systems, distinguishing them from a randomly reacting chemical soup and necessary for building the higher complexity levels. Attempt at defining life is a reflexive activity of life. As representants of this type of structure, we are of course familiar with life, but from a very objective physical viewpoint, one must acknowledge that its remains puzzling. Life is self-organized, self-invariant notwithstanding material renewal and environmental changes (Cornish-Bowden and Cárdenas, 2008), self-maintained away from equilibrium, self-replicating and to some extent self-evolving. These numerous characteristics explain the diversity of the topics addressed in the literature on the origin of life. A widespread expectation is that insights into the secrets of life could be provided by the comparative composition of the living and inanimate matter. Many molecules are indeed different between the living and mineral worlds but the idea turned to be not sufficient. The existing biomolecules result from fortuitous evolutionary circumstances and could have been different. For example, in eubacteria and eukarya, similar responses to heat stress are mediated by completely unrelated molecules (sigma factor switch or HSF respectively). This relative arbitrariness in the choice of molecules, is also suggested by anecdotic findings such as for example (if confirmed), variant DNA molecules of exotic cells with arsenic instead of phosphate (Wolfe-Simon et al., 2010). Resembling biological structures can also be generated by unrelated machineries, through a phenomenon of convergence driven by physical constraints. The main organizational molecules are now proteins, but this role is believed to have been ensured in the past by polyribonucleotides (Gilbert, 1986). In the same way, if life exists in other planets, it could use different molecules and perhaps different modes of information archiving. Attempts at reconstituting the onset of life from the biogeological evolution, listed for example in (Vaneechoutte and Fani, 2009), are essential for understanding terrestrial life, but could be an impasse to define life per se. Given that life cannot be defined from its constituents, it could rather emerge from the relations existing between them. In this regard, life would rejoin mathematics and physics. Following Georg Cantor, mathematics deal only with the relationships between objects, rather than on their precise nature. The same principle holds in physics and particularly in statistical mechanics, for which very precise macroscopic predictions can be derived from the simplistic assimilation of the constituents as hard spheres (Lebowitz, 1993). To extend this principle to life, let us examine the more general definition proposed as soon as in 1886, long before molecular genetics, by the visionary physicist Ludwig Boltzmann: "The general struggle for existence of animate beings is not a struggle for raw materials - these, for organisms, are air, water and soil, all abundantly available - nor for energy, which exists in plenty in any body in the form of heat $Q$, but of a struggle for entropy $S$, which becomes available through the transition of energy from the hot sun to the cold earth" (Boltzmann, 1886). The same view was recovered by Erwin Schrödinger, who envisioned living matter as molecular organizations capable of decreasing local entropy and which thereby "evade the decay to equilibrium" (Schrödinger, 1944). Since life is rooted in the chemical world, this view suggests that some chemical discernment can emerge from molecular motions in nonequilibrium conditions, but beyond this general principle, these physicists did not address the precise mechanisms by which free energy is used for cellular organization. In the same way, statistical entropy is sufficient for predicting organizational benefits, but does not reveal the user guide of life. The present study is aimed at reviewing and classifying the elementary statistical mechanisms which concur to render biochemical systems "intelligent". A classical way to achieve this end and conforming with the second law of thermodynamics, is based on dissipative cycles with net fluxes. Selected examples will be presented to show that these 
recipes depend on non-equilibrium steady states maintained at the expense of matter and energy. But an other way results from statistical biases generated in a non-dissipative manner, through equilibrium cooperativity. The representative examples of organizational strategies reported in this study adopt the Boltzmann's view, based on the statistical treatment of ensembles of stable and distinguishable particles.

\section{Organization and time flow come from one-way doors}

As it is obviously not judicious to build a barrage across a closed lake to produce electricity, conversely the generation of net fluxes requires supplies of exogenous matter or energy. Homogenous thermal agitation represents a high internal energy which is abundant in warm cells, but according to the Boltzmann's statement quoted above, is not exploitable to generate directional fluxes (If it was possible, "thermosynthetic" life would certainly exist, which is not the case). Life is not a closed lake but necessarily an open system, in which the importance of irreversible reactions has long been pointed (Atkinson, 1977). A starter kit for the rapid development of the following concepts is first proposed, in the form of a mechanical model which, though amusing, will prove relevant and singularly appropriate to illustrate the primary modes of biochemical discernment.

\subsection{Rebuilding statistical dynamics in a camembert box}

One should remain aware that drawing one-way arrows in biochemical schemes has important consequences. A mechanical system made of hard spheres moving in a camembert box, is proposed here to illustrate some basic principles (Fig. 1). The temperature, volume, pressure are considered as roughly constant, as in biological systems. The box is divided into 3 space units A, B, C, separated by walls pierced by gates and it is submitted to random shaking to mimic thermal agitation. This system can also be envisioned in 3 dimensions without need for shaking, if replacing the box by an aquarium and the spheres by fishes randomly swimming and crossing the gates following a Poisson law (joke in French). The states of the system are defined only in term of position of the spheres in these compartments (neglecting their position inside each compartment). Considering the symmetry of this system (Fig. 1a), it is obviously expected that the presence of the ball in every compartment is equally probable.

To illustrate the capacity of a one-way flux to decrease entropy and its consequences, let us insert a one-way door between the compartments $\mathrm{B}$ and $\mathrm{C}$, whose opening is triggered by transfer of some translational and rotational energy of the ball passing from $\mathrm{B}$ to $\mathrm{C}$ (Fig. $1 b, c)$. It can be reasoned that the probability for the ball to be in $\mathrm{C}$ is slightly increased by the presence of the one-way door, because there are 2 ways to enter $\mathrm{C}$ but only one to escape it. Conversely, the probability to be in B, from which there are two ways to exit and one to enter, is decreased. Reducing the accessibility to certain possible microstates decreases the uncertainty about the position. This phenomenon meets the notion of statistical entropy and can be quantified using an adimensioned version of the Gibbs entropy $\left(S / k_{B}\right)$ adjusted to a single particle with $n$ possible states, by using a logarithm whose base corresponds to this number:

$$
H=-\sum_{i} P_{i} \log _{\mathrm{n}} P_{i}
$$

Example: for $n=3$ corresponding to a "trit", equivalent to the bit of binary states, 
$H=-3\left(\frac{1}{3}\right) \log _{3}\left(\frac{1}{3}\right)=1$

For $N$ identical and independent balls, each one with the 3 possible locations, we have $H=N$. This abstract concept of probability can be concretized by increasing the number of balls, assuming the intuitive notion of ergodicity which encloses that a probability for one ball is equivalent to a proportion for many independent balls. Common sense suggests that the frequency of the transitions from one compartment to an adjacent one, is proportional to the number of balls in the starting compartment. Hence, the same number of balls is expected on average in every compartment in Fig. 1e, while with the one-way door (Fig. 1g), we have $2 a$ $=b+c, a=2 b$ and $a+b=c$, which gives the following proportions:

$\mathrm{P}(\mathrm{A})=a /(a+b+c)=1 / 3$,

$\mathrm{P}(\mathrm{B})=b /(a+b+c)=1 / 6$

$\mathrm{P}(\mathrm{C})=c /(a+b+c)=1 / 2$

Hence,

$H=-\left[\left(\frac{1}{6}\right) \log _{3}\left(\frac{1}{6}\right)+\left(\frac{1}{3}\right) \log _{3}\left(\frac{1}{3}\right)+\left(\frac{1}{2}\right) \log _{3}\left(\frac{1}{2}\right)\right]=0.92$

Entropy has expectedly been lowered by the presence of the one-way gate, from 1 to 0.92 . Consequently, the flux of balls passing from $\mathrm{C}$ to $\mathrm{A}$ is now higher than from $\mathrm{A}$ to $\mathrm{C}$ : the principle said of micro-reversibility is broken. Since the number of balls differs between the compartments $\mathrm{A}$ and $\mathrm{C}$, despite an equilibrium constant of 1 (the door between $\mathrm{A}$ and $\mathrm{C}$ has of course the same width in both directions), a free energy appears, which would be for a chemical system (Beard and Qian, 2007):

$\Delta G_{(C \rightarrow A)}=-k_{B} T \ln \frac{3}{2}$

where $\mathrm{T}$ is the degree of agitation of the balls. These simple considerations are sufficient to explain many aspects of cell biology based on gradients of chemical concentration across membranes, such as the electrical nerve conduction, or the synthesis of ATP coupled to the release of a proton gradient across a mitochondrial membrane. It will be shown that these principles are also at work in absence of compartments. In this case, the states of molecules are not their spatial localization but their conformational or binding states.

\subsection{Micro-reversibility, -irreversibility and the second law}

The principle of micro-reversibility connected to the second law, is the rule at equilibrium (Tolman, 1925). It implies that the passive one-way door described above (Fig 1b, c, g), has no equivalent in the real chemical world. One-way permeable membranes would break the second law of thermodynamics which stipulates that the extraction of directional fluxes from random thermal agitation is impossible, as suggested in the example of the barrage across a closed lake and as clearly stated in the assertion of Boltzmann quoted in the introduction. This phenomenon is statistical and related to the fact that macroscopic systems spontaneously evolve from less probable to more probable states. For example, the gathering of the entire population of balls in the same compartiment of the camembert box (Fig. 1f) appears intuitively less probable than dispersed balls (Fig. 1e). The probability of a macrostate is the proportion of microstates underlying it. Consider the simple example of dice gambling. When 
throwing 2 dices, there are 6 times more chances to obtain 7 than 2, because 7 is a scattered macrostate corresponding to 6 microstates $(1-6,2-5,3-4,4-3,5-2$ and 6-1) while 2 is an unscattered macrostate corresponding to only one microstate (1-1). Unscattered macrostates become less and less likely when increasing the number of system constituents. The excess of scattered macrostates exponentially increases when increasing the number of dices. For $n$ dices, the chance to obtain $\mathrm{n}$ is only $1 / 6^{n}$. This amplification of the microstate number is handable by using its logarithm in the definition of entropy. Following the example of dice gambling, it is obvious that a macrostate is all the more probable that the underlying microstates are numerous, even if these microstates are not observable. Indeed certain microstates, such as 3-4 and 4-3, can be distinguished only if the dices are identifiable (for example thanks to different colours). The distinction between microstates is of course impossible for real systems made of identical particles, but in the Maxwell-Boltzmann statistics, they undoubtedly exist. This assumption is not generalizable to all objects in physics, such as bosons which obey to different statistical rules.

\subsection{The time's arrow, memory and ageing}

The present time is the frontier between the past and future and has no intrinsic dimension. The future is also conceptual only. Even sets of deterministic differential equations give at best approximate promises since they cannot be exhaustive enough and grounded on known initial conditions. Hence, among the connected notions of past, present and future, only the past can be concretized in the form of its memory. But given that the past can be recorded only at the expense of some increase of entropy, the notion of time concerns only organized systems because those which have reached equilibrium cannot increase entropy further. The stable elementary matter components are memoryless, timeless and not subject to ageing. An homogenized dynamic system made of such components also lost memory, consistent with the absence of time in the units of equilibrium constants. In turn, systems out of equilibrium can spontaneously evolve. The evolution time scale depends on the equilibration rate of phenomena, but the orientation of the time arrow is always the same.

It is worth noting that this time's arrow is not embodied in the basic laws of physics which are compatible with a possible reversibility of time. This is true for Newtonian physics, kinetic theories of molecular systems, as well as in the description of the quantum world. Time irreversibility is intimately connected to the second law (Lewis, 1925; Lebowitz, 1993) and seems to arise somewhere between micro and macrostates. Our feeling of time irreversibility is anchored in a so usual experience, that an easy way to determine if a dynamic system is time-reversible or not, is to appreciate its strangeness in a film reeled backward. A system at equilibrium is clearly time-reversible (Fig. 1a and e) since its forward and backward evolutions appear equally normal. By contrast, if run backward, a film of Fig. 1c would appear strange since the door would open before the contact with the ball. Hence, this cannot be an equilibrium. In the same way, if one tries to order in time the panels e and $f$ of Fig. 1, common sense strongly suggests that the snapshot Fig. If precedes Fig. 1e, because it seems more organized and looks like a forced initial condition. In fact, if one could distinguish every individual ball, the two panels represent equally probable microstates (probability $\mathrm{P}=1 / 3^{n}$, where $\mathrm{n}$ is the number of balls). The illusion that the left one is more likely at equilibrium, results from the fact that it resembles many other ones, by interchanging the balls and/or the compartments. It is said more typical. This good intuition becomes misleading in case of confusion between micro and macrostates, for example if one supposes that the lotto combination $1,2,3,4,5,6$, is less likely than $4,13,27,29,34,40$. Both have exactly the same probability to occur (but betting on the first one is perhaps preferable to not share the gain in case of success). Microstates with scattered balls are permutable and thus much more 
numerous than those with clustered balls. This explains why the transition between organized and disorganized macrostates appears irreversible.

But the reversal of macrostates is not strictly prohibited. Suppose that the snapshot of Fig. 1e derives from Fig. $1 \mathrm{f}$ and that it is used as an initial condition by inverting exactly all the individual velocities (from the friction-induced motions in the close surrounding, to the infinitesimal cosmological perturbations); then, Fig. If would in theory be recovered. But in fact, supposing that the sequence of events between the snapshots of Fig. If and Fig. 1e could be inverted, is an artificial twist to correlate the balls. From the Boltzmann's concept of uncorrelated particles in statistical mechanics, the second law is not an absolute physical law but only a probability, complementary to a formulable and verifiable probability to be violated (Wang et al., 2002). This phenomenon is trivial when decreasing the scale: the Brownian motion for example, can appear as a succession of micro-violations of the second law (but which are useless at the macroscopic level given that they are not oriented in the same direction). Using this assumption, Fig. 1f can be obtained by chance. The optimistic conclusion of these considerations is that our ageing is not obligatory but just a probability. A more deceptive one is that at our scale, this probability is so infinitesimally close to 1 that it is in practice a certainty. Is it possible that the concrete irreversibility of ageing could be dictated by these abstract probabilistic considerations? Why not, if one remembers the perceptive assumption of James Clerk Maxwell that "the true logic of this world is in the calculus of probabilities".

Certain concerns raised about an entropic origin of the time's arrow result from a misconception of the name "information entropy", suggesting that the ignorance of the observer is in some way involved in the genesis of time. In this respect, the word information is misleading and should preferably be replaced by other terms such as permutational, configurational, statistical or, preferably, probabilistic, if one accepts probability as a genuine physical entity. Indeed, even the exhaustive knowledge of all individual microstates (if possible) would not prevent things to evolve from less probable to more probable macrostates. The time's arrow of the universe preexisted before observers and allowed their emergence. Intriguingly, it has also been proposed that the arrow of time is generated by symmetrybreaking phenomena occurring far from equilibrium. But by itself, the mere postulate of an environment far from equilibrium implies that the arrow of time preexisted before the symmetry breaking events. By climbing up the cascade of causal arrows, we are brought to conclude that the master arrow, to which all the secondary ones are unidirectionally subordinated, is the big bang (section 7).

\subsection{The probabilistic root of entropy}

Leon Brillouin and Edwin Jaynes pointed the inner equivalence between the entropy of the kinetic theory and that of information (Brillouin, 1956; Jaynes, 1957). If returning to an absolute entropy using the natural logarithm and if the $\mathrm{W}$ possible states of the system are all equivalent, then

$H=-\sum_{i} P_{i} \ln P_{i}=-W \frac{1}{W} \ln \left(\frac{1}{W}\right)=\ln W$

which is the Boltzmann equation if reconnected to energy through the Boltzmann constant $k_{B}$. The purely probabilistic and dimensionless definition of entropy will be preferred in the present study. The statistical nature of entropy makes it applicable to any objects, beyond the sole field of physical chemistry. By construction, the entropy of juxtaposed independent systems is additive. The logarithm, which can appear no more than a formal tool, is rational if considering that it is the function that transforms the multiplications into additions. This conversion is the very basis of probabilities given that the probability of a sum of independent 
events, is the product of their individual probability. The increase of entropy is often considered to result from a dispersal of energy, which remains globally constant in an isolated system but tends to spread over the maximum number of microstates. If temperature is of course necessary for molecular dynamics, the statistical expansion of microstate configurations is expected without other physical condition, consistent with the absence of dimension of probabilistic entropy. If eliminating the one-way door in Fig. 1g, the excess of balls in the space unit $\mathrm{C}$ would disappear, with a rate depending on the degree of agitation of the balls. In parallel, the entropy, as defined in Eq. 2.1, is maximized and the subsequent snapshots would resemble more to Fig. 1e. The issue becomes peculiar for one ball since snapshots can no longer be used to appreciate the scattering of a single ball. One can derive that the fraction of time spent by the ball in each compartment equalizes; but defining the time's arrow by measuring time seems somewhat strange. In such a situation, the purely probabilistic nature of entropy clearly appears as the unique explanation. This probabilistic entropy of a single ball is interesting because it is at first glance cleared from the problems of possible correlations and resonance between the different interacting particles, opposed to the Boltzmann's theory.

\section{Maintenance of one-ways doors and organization in biochemical systems}

\subsection{Biochemical one-way doors are generated and maintained by matter and free energy flows}

One-way transitions in biochemical cycles, similar to the one-way gate in the camembert box in section 2, can be generated by two means, by inputs of exogenous energy and matter (Fig. 2). A variety of sources of free energy is available for this purpose (Kaufmann, 2009). The import of free energy and dissipation of degraded energy is the admitted fuel of life but the precise mode of energy delivery should be clarified.

- $\quad$ Energy delivery. The primary source of energy is provided by organic molecules with Gibbs energy deriving from food intake for animals, or photosynthesis for plants. Fragments of these molecules are used in the mitochondria to generate a gradient of proton concentration across a membrane, like a water barrage. The release of this potential energy is coupled to the $\mathrm{F}_{0} \mathrm{~F}_{1}$-ATP synthase (equivalent to a hydroelectric turbin) converting the proton-motive force into the generation of adenosine triphoshate (ATP), the money of energy selected in all forms of terrestrial life.

- Why ATP can work as a money of energy. This is not because of some magical "energy-rich bond" sometimes found in biochemistry courses, but for the following conjunction of reasons:

(i) ATP contains, as many other molecules, a fragile (but not very energy-rich) chemical bond between the $\beta$ and $\gamma$ phosphates, whose breakage is exergonic and generates ADP.

(ii) A high ATP/ADP concentration ratio in the cell, making the ATP to ADP conversion micro-irreversible in practice.

(iii) The existence of a remarkable protein domain capable of splitting ATP, the ATPase domain, evolutionary shared out in many different proteins in the cell. In this respect, the fact that the earliest complementary genes encode ATP/GTP binding and ATPase domains (Trifonov, 2009), could be not fortuitous.

Together, these instruments allow to easily deliver units of external free energy to elementary transitions in the internal biochemical network. 


\subsection{Persistence of organized systems}

Once an organized structure is built by exporting entropy in the surroundings, it is hopefully expected to survive for sometime. Every organized thing around us will disappear one day and perhaps be replaced. A window for example, would be much more stable when crumbled on the floor. If so, why things do not degrade, vanish or burn immediately to their maximal entropy state, as predicted by the second law? The reason is purely kinetic. We don't know exactly when the window will crumble because fortunately a barrier, called activation energy, prevents it to explode immediately. This activation energy can be provided by throwing a stone in. Similarly, the time necessary for biochemical reactions to proceed, even when exergonic, is generally incompatible with life, and should be strongly shortened by proteins called enzymes, which increase the probability of efficient molecular collisions (with appropriate orientation, position of reactive residues etc.).

\subsection{Now, the main organizational molecules of life are proteins}

The one-way transitions and microstate suppressions described in the following sections are mediated by a series of properties of macromolecules including:

- Stereo-specificity. The tridimensional structure of proteins allows specific interactions (such as the "lock-and-key" model) with other molecules.

- Induced or spontaneous deformability, a property involved for example in the "induced fit" and "conformational selection" mechanisms.

- Allostery, or the capacity of molecules to change their shape upon ligand binding or chemical modification (such as phosphorylation).

- Assymmetry. Chirality is generalized in biochemical structures, such as proteins, from their constituent amino-acids to their global folding.

- Memory, related to slow conformational relaxation following deformation.

All these roles are now mainly ensured by proteins in terrestrial organisms, but as proposed above, other types of molecules could have been selected on the basis of these properties. The large information content, equivalent to negative entropy or negentropy (Brillouin, 1956), which accumulated in certain macromolecules during evolution, is sometimes sufficient to generate asymmetric and cooperative behaviours in a micro-reversible manner (section 5), as illustrated by the saturation of multimeric allosteric proteins and the nonlinear combinatorial occupation of DNA regions by regulatory proteins. However, many examples show that micro-irreversible processes with one-way transitions, remain a major source of steep sigmoidicity and discernment.

\subsection{Dosage and accuracy of biological responses: hyperbolic vs sigmoidal responses}

A typical low entropy process is the sigmoidal response. Saturable linear responses obtained at equilibrium are non-sigmoidal and have no intrinsic discrimination capacity (Fig. 3), while sigmoidal ones are involved in switches, thresholds and ultrasensitivity (Koshland, 1987). A response curve can be a sigmoidal function of several parameters, such as the concentration of signal molecules (ligands, substrates), or their relative propensity to associate or dissociate. This latter case has a widespread but overlooked importance for the specificity of biochemical reactions in vivo, in the mechanism of substrate selection described in section 4.1. Although sigmoidal responses are generally assumed to result from signal amplification, they are often due to the reduced effect of low signals. This is the case in the examples listed in this report, such as for kinetic cooperativity (section 4.3), in which high affinity binding sites become prominent only at high ligand concentration. The following examples will show that 
sigmoidal response curves emerge from macromolecular state cycles with micro-irreversible transitions, and are direct consequences of the decrease of statistical entropy. The King and Altman steady state treatment (King and Altman, 1956) clearly illustrates how one-way transitions eliminates certain diagrams compared to equilibrium, thereby allowing the genesis of sigmoids by forbidding fraction simplifications otherwise possible.

\section{Dissipative cooperativity}

In this section, typical examples are listed to illustrate mechanisms allowing to bias statistical state representation and to increase biochemical discernment at the expense of energy and matter.

\subsection{Dynamic discrimination between wrong and correct, but resembling molecules}

This example of molecular discernment combines all the notions introduced earlier: the involvement of a one-way door, the need for free energy supply and a sigmoidal reaction curve, for a powerful improvement of the specificity of chemical reactions. Molecular interactions in the cell depend both on the facility for molecules to collide conveniently (association rate), and on the longevity of the association (dissociation rate). The ratio of these two rates determines the time-independent equilibrium constant or affinity. Biological reactions begin with a molecular association event, but the specificity of this first step should be secured. The active site of anabolic enzymes can accommodate different molecules present in the cell, sometimes with equivalent binding rates. This problem becomes acute for polymerases such as DNA polymerases, RNA polymerases and ribosomes, which should respect the genetically-programmed sequence of residues encoding life informations and avoid the synthesis of harmful aberrant proteins. In the example of translation, John Hopfield (Hopfield, 1974) and Jacques Ninio (Ninio, 1975) were intrigued by the low error rate of the ribosome in vivo, which contrasts with the relatively low affinity of loaded tRNA for polysomes observed in vitro. These authors proposed a mechanism which strongly amplifies the capacity of discrimination between the twenty amino acids. At each elongation step, the polysome can admit all tRNA-amino-acids with nearly the same association rate, so that the discrimination can occur only at the level of dissociation. Hence, a first necessary condition to be satisfied to avoid the immediate erroneous fixation of inadequate amino-acids to the nascent protein, is that their covalent ligation of should not be too rapid relatively to their rates of interaction. If the binding rates of the resembling substrates are the same and follow the classical Michaelis-Menten reaction, it can be shown (appendix 10.1) that even in this favourable condition $\left(k_{c}<<k_{d}, k_{d}^{\prime}\right)$, the difference of fixation of a correct substrate compared to a wrong one, does not exceed the ratio of their respective dissociation rates. Since this ratio is insufficient to explain the observed biological specificity, Hopfield and Ninio proposed that the quality of the substrates is further discriminated through an additional mechanism based on the combination of two ingredients: (i) the previously postulated time scale separation between the processes of non-covalent interaction and of covalent ligation of the substrate, and (ii) an energy-driven one-way transition whose frequency is written below $k^{*}$ (for example through hydrolysis of GTP for translation or NTP for polynucleotide polymerisation). The stronger this $k^{*}$ transition is driven, the less probable is the direct ligand rebinding to yield ES* (Hopfield, 1974). Using these conditions, the sigmoidal behaviour of this substrate selection mechanism can be evidenced by the global reaction rate. If considering (i) that the catalytic step is infrequent relatively to the other reactions; (ii) that the two dissociation steps have the same constant $k_{d}$, then, the average reaction rate can be obtained 
using a triangular circuit with one-way doors (Fig. 4a). The resulting rate equation, takes the form:

$k_{R}=\frac{A}{B+C k_{d 2}+k_{d 1} k_{d 2}}$

which is a decreasing sigmoidal function of $k_{d 1}+k_{d 2}$. The capacity of a substrate to be fixed is critically dependent on the balance between the activation reaction $\left(k^{*}\right)$ and the stability of the substrate $\left(k_{d 2}\right)$. If $k_{d 2}>k^{*}$, the ES* state is quasi-inexistent and the reaction is impossible. If substrate association survives the $k^{*}$ transition $\left(k_{d 2}<k^{*}\right)$, then $\mathrm{ES}^{*}$ is more stable and the covalent ligation can proceed.

The efficacy of this mechanism can be quantified using the example of two competing substrates present in similar concentrations. With the Michaelis-Menten reaction, the discrimination ratio $\mathrm{R}$ between the wrong $\left(\mathrm{S}^{\prime}\right)$ and the correct $(\mathrm{S})$ substrate, is the ratio of their dissociation rates $k_{d} / k_{d}^{\prime}$ (appendix 10.1). Dissociation rates $\left(k_{d}\right)$ are Poisson parameters, so that the probability to remain associated exponentially decrease with time according to $\exp \left(-k_{d} t\right)$, but the mean waiting time is simply $1 / k_{d}$. A recognition of wrong and correct substrates only based on this ratio of waiting times is insufficient to explain the low error rate of polymerisation processes. The increase in discrimination in the Hopfield-Ninio mechanism can be determined using the scheme of Fig. $5 \mathrm{~b}$. In this scheme, the rate of productive substrate binding $k_{T}$ is

$k_{T}=\frac{k_{U} k_{a}[S]}{k_{U}+k_{d 1}}$

with

$k_{U}=\frac{k_{c} k^{*}}{k_{c}+k_{d 2}}$

hence

$k_{T}=\frac{k_{c} k^{*} k_{a}[S]}{k_{d 1} k_{d 2}+k_{c}\left(k_{d 1}+k^{*}\right)}$

If $k_{c}<<k_{d}, k^{*}$, the ratio of discrimination between the correct and wrong substrates is

$R=\frac{k_{T\left(S^{\prime}\right)}}{k_{T(S)}}=\frac{k_{d 1} k_{d 2}}{k_{d 1}^{\prime} k_{d 2}^{\prime}}$

$\mathrm{R}$ is now the product of two dissociation rate ratios, consistent with the fact that while the error rate expected from the simple dissociation rates is only $10^{-2}$, that actually observed is around $10^{-4}$.

4.2. Breaking or not breaking induced fit associations: the choice between molecular complexes and highly specific dynamic interactions

Non-covalent multimolecular structures are numerous in the cell (such as ribosomes, RNAPII etc) and can be built by stepwise assembly of components such as: $\mathrm{A}+\mathrm{B} \rightleftharpoons \mathrm{AB},+\mathrm{C} \rightleftharpoons$ $\mathrm{ABC},+\mathrm{D} \rightleftharpoons$ etc. In equilibrium conditions, such a chain leads to the molecular trapping of the 
early components ( $\mathrm{A}$ or $\mathrm{B}$ in this scheme), rendered prisoners in the complexes by the "capping" effect of the late components ( $\mathrm{D}$ and following ones). But in addition to macromolecular complexes whose constituents are expected to remain associated, cellular function and responsiveness to changing conditions also necessitate continuous collisions between soluble macromolecules moving in water. Since binding specificity and dynamics are mutually conflicting, the maintenance of this dynamic interactome requires the involvement of active dissociation mechanisms breaking equilibrium. Precisely, the crux of the biochemical discernment mechanisms described in sections 4.3 and 4.4, is the active dissociation of molecular complexes. It concerns a widespread mode of molecule assembly called induced-fit, symmetric to the phenomenon of conformational selection (Hammes et al., 2009; Weikl and von Deuster, 2009). Very specific molecular interactions can be obtained from weak primary interactions, through the process of induced fit proposed by Daniel Koshland (Koshland, 1958), in which, after a shaky initial association, the shape of a macromolecule progressively adjusts to that of the ligand by increasing the number of chemical bonds. This mechanism allows reaching precise molecular stereo-adjustments between weakly preorganized but supple macromolecules. The induced fit mechanism has been invoked for enzymes with lid-gated sites (Sullivan and Holyoak, 2008) or for DNAbending proteins such as IHF (Khrapunov et al., 2006; Sugimura and Crothers, 2006). In the two-step mechanism proposed in these studies, IHF binds first to linear DNA, which then progressively bends around IHF. The situation is more controversial for the eukaryotic DNAbending protein TATA-binding protein (TBP). The bending of its target DNA motif, the TATA-box, could follow an initial association of TBP with linear DNA (Zhao and Herr, 2002), but alternatively DNA bending and binding have also been proposed to occur simultaneously (Masters et al., 2003). The kinetic rational of simultaneous binding and folding has however been questioned (Hammes et al., 2009). In theory, induced fit binding can participate to a dynamic cyclic process if it is coupled to conformational selection (Fig. $6 \mathrm{~b}$ ). In this latter mechanism, the role of the ligand is not to impose the shape of the macromolecule by contact, but to bind to and stabilize a particular pre-existent tridimensional state. The conformational selection mechanism is also conceivable for TBP, whose fixation to the TATA box is stimulated by DNA pre-bending (Parvin et al., 1995; Vichi et al., 1997).

Examination of Fig. 6b,c,d suggests that the induced fit mechanism can remain dynamic only if it is moderate. Indeed, there is no rational reason that a ligand molecule can escape a macromolecule tightly moulded to its shape. Conversely for the conformal selection aspect, it is unlikely that a macromolecule spontaneously folds into a high energy conformation, finely pre-adapted to a ligand which is absent. The neglectable reverse-induced fit reaction is illustrated by the very stable association observed between TBP and DNA, exceeding several hours in vitro, suggesting that in practice, TBP is trapped in DNA. The extreme degree of specificity reached upon induced fit appears, at first glance, free of thermodynamic charge; but in fact, it should be paid for maintaining interaction dynamics. Indeed, thermally driven fluxes can no longer take place between conformational selection and induced fit if the associated stabilization is too tight. The induced fit mechanism is particularly appropriate for the accretion of multimolecular complexes but conversely, it is difficult to envision this phenomenon as a part of a dynamic cycle. Besides, claiming that micro-reversible binding cycles follow an induced fit rather than a conformational selection mechanism, is poorly relevant since these two processes are mutually constrained in a closed cyclical system, following the Wegscheider relation (appendix 10.2). Interactions are all the more freezed that the energy drop during the induced fit step is important. This is clearly illustrated by DNA-bending proteins which would remain indefinitely trapped in DNA once the maximal number of chemical bonds is reached. Hence, non-equilibrium mechanisms should intervene to unblock the system and render the waiting time before dissociation 
compatible with cell physiology. Two forced dissociation mechanisms exist: (i) the active displacement of the ligand and (ii) the chemical transformation of the ligand into a product with a different structure, leading it to fall off the enzyme binding site. Consistent with section 3.1 , these mechanisms depend on supplies of either energy or fresh substrates respectively. The energy-driven displacement of a ligand is concretely illustrated by the interaction between TBP and the TATA-box, which appears nearly unlimited in time in vitro but is rapidly cancelled in vivo, by ATPases such as Mot1 in yeast. An important situation of induced fit breaking is encountered in the case of kinetic cooperativity (following section).

\subsection{One-way doors generated by macromolecule memory, allow to avoid indesirable reactions: the example of glucokinase.}

Small molecules are rigid and poorly deformable and their relaxation time is so rapid that their conformational memory should be astonishingly short-term. By contrast, big proteins can take a longer delay to recover their ground state following conformational perturbations. This allows them, for example, to remind the shape of a ligand bound in the recent past, thereby facilitating the access of subsequent ones. As explained previously (section 2.3), memory is the mark of low entropy systems which have discerning properties. A wonderful example of discerning molecule is glucokinase, an enzyme essential for regulating glucose consumption in the organism.

The glucose sugar is the predominant source of carbon and energy for most cells in multicellular animals. The first step of its transformation is its phosphorylation into glucose-6phosphate, which is catalysed by hexokinases and leads to glucose consumption. These cellular enzymes work as soon as glucose is available. Certain tissues, such as liver and pancreas, contain a variant type of hexokinase (hexokinase D also named glucokinase), which catalyses the same reaction, but in a glucose concentration-dependent manner (Cárdenas et al., 1979; Cornish-Bowden and Cárdenas, 1987). Liver has the unique capacity to store the surplus of glucose into glycogen and to do that only when the concentration of circulating glucose is judged higher than necessary for the immediate energetic needs of the organism. Hence, liver glucokinase is active over a certain threshold of glucose concentration, but not when glucose is limiting. In the pancreas, the glucose concentration-sensor role of glucokinase is used to determine when it is time to secrete insulin, to limit the amount of circulating glucose. Moreover, glucokinase can take into account other informations such as ATP concentration.

This regulation of glucokinase activity would have required at our level, an advisory office well informed by a measurement service. But at the molecular level and in nonequilibrium conditions, this refined discernment depends on the sole responsibility of a single molecule. Glucokinase is conformationally re-shaped upon glucose binding through an induced-fit mechanism (Heredia et al., 2006), so that it can recover its unliganded form after glucose removal. Hence, the key mechanism allowing this regulation is the microirreversibility of the catalytic step (rate $k_{c}$ in Fig. 6f), which can easily be conceived if the tridimensional shape of the reaction product is different from that of the substrate, leading to its micro-irreversible departure. As explained in section 4.2, an equilibrium system mixing the mechanisms of conformational selection and induced fit, would be no longer dynamical in case of strong induced fit stabilization $\left(k_{i}\right)$, by forbidding ligand escape. But when relieved from the equilibrium constraint, the system can cumulate different features (Fig. 6f): (i) $k_{i}$ can be strong enough to forbid the reverse reaction $\left(k_{-i}\right)$ and the spontaneous dissociation of the substrate from E'S $\left(k_{d}^{\prime}\right)$; (ii) reciprocally, the relaxation rate $\left(k_{r}\right)$ is high once the substrate has been flushed out, and its reverse transition $\left(k_{-r}\right)$ can be neglected, since it would correspond to the very improbable spontaneous folding of the enzyme into a form $E^{\prime}$ pre-fit to an absent 
ligand. Using these conditions, the substrate concentration-dependent reaction rate can become sigmoidal (Fig. 4b) (Min et al., 2009).

Beside the technical treatment of this system, it is more important to conceive its underlying principle, which allows that large differences of enzyme reactivity are obtained in response to small differences of substrate concentration. This phenomenon results from a competition between the relaxation of the enzyme $\left(k_{r}\right)$ and substrate rebinding $\left(k_{a}^{\prime}[S]\right)$. Over a certain substrate concentration threshold, the substrates always access to the enzyme in a state (E'), ideally shaped for reacting, so that the enzyme is locked at maximal activity and prevented to relax (Rabin, 1967). Conversely, glucokinase poorly works at low glucose concentration since the enzyme has time enough to recover its ground state after every catalytic reaction. If $k_{r}$ is low relatively to $k_{a}^{\prime}$, then the sigmoidal shape becomes steeper, as shown in the numerator of the rate in Fig. 4b. A considerable property of kinetic cooperativity is that it is possible for monomeric enzymes, contrary to spatial cooperativity in multimeric proteins described in section 5. Indeed, according to the suggestion of Edward Whitehead (Whitehead, 1970), there are two modes of cooperativity: through space and through time. The latter case, to which glucokinase belongs, is obtained when a single object cooperates with itself, or more precisely with its future state, thanks to the memory of the recent past inscribed in its conformational resilience. Kinetic cooperativity can be evidenced in steady state enzymatic reactions but not in ligand binding isotherms. Consistently, when deleting the catalytic activity of a kinetically cooperative enzyme, the substrate saturation curve reverts to the classical equilibrium hyperbola (Lin and Neet, 1990). Studies of kinetic cooperativity are so far restricted to enzymatic reactions but they could be extended to other situations. For example, while the presence of multiple binding sites in DNA allows spatial cooperativity, the involvement of irreversible steps in the course of promoter saturation, also makes possible kinetic cooperativity, if the system is open and refuelled with matter and energy.

\subsection{Example of detailed balance breaking in the control of gene expression}

The necessity to break equilibria does not concern only the cycle mixing conformal selection and induced fit described above, but also linear binding chains. Hierarchical occupation of DNA by TFs can occur in equilibrium (Fig. 7a), but often requires equilibrium-breaking machines. The example proposed here is the hierarchical occupation of DNA by transcription factors (TFs). DNA-binding cooperativity between TFs is generally examined at equilibrium (Bolouri and Davidson, 2002; Bintu et al., 2005; Michel, 2010). The influence of nucleosome position on the regulation of gene expression is also generally envisioned under the equilibrium perspective (Segal and Widom, 2009; Mirny, 2010). But this view omits certain energy-driven steps in the course of promoter saturation by TFs. This is the case for the hierarchical occupation of DNA mediated by chromatin remodeling. A typical illustration of this mode of regulation is the Mouse Mammary Tumor Virus (MMTV) promoter, which is activated by fixation if the TF glucocorticoid receptor (GR), when it is itself activated by glucocorticoid stress hormones such as cortisol. The triggering action of GR can then be enforced by a secondary group of TFs including NF1, Oct-1 and Oct-2. This hierarchical action is due to the differential capacity of these TF to access DNA. GR can binds to its DNAbinding site (GRE) even when it is in close contact with a nucleosome, contrary to NF1 and Oct TFs, which should encircle the double helix (Eisfeld et al., 1997). But DNA-bound GR recruits a chromatin-remodeling enzyme of the SWI/SNF family called BRG1 (Fryer and Archer, 1998), which can displace the nucleosome in an ATP-dependent manner, thereby opening the way to NF1 and Oct (Fig. 7b)(Archer et al., 1992; Chávez and Beato, 1997). When envisioned under an equilibrium assumption, this hierarchical mechanism, which can be written $\mathrm{D} \rightleftharpoons \mathrm{D}-\mathrm{GR} \rightleftharpoons \mathrm{D}-\mathrm{GR}-\mathrm{NF} 1$ (where $\mathrm{D}$ is the MMTV DNA promoter), implies that 
GR is prevented to dissociate as long as NF1 is present on DNA. This trapping phenomenon has not been observed experimentally. Indeed, these studies were then contradicted in following articles which reported an inverse hierarchy in which NF1 and Oct-1 preset chromatin favouring GR binding (Belikov et al., 2004). Such reciprocal influences between GR and NF1 for binding to the MMTV promoter were called dualistic (Hebbar and Archer, 2007). The inconsistencies between these observations can be ruled out by breaking the equilibrium. This can be achieved by the energy-dependent activity of chromatin-remodeling enzymes. The rational analysis of this system reveals an exquisite mechanism of cooperativity, allowing to finely tune the responsiveness and sensitivity of gene expression in response to non-physically interacting TFs (Michel, 2011). It allows to render the action of the hierarchically-dependent TFs strongly overadditive (Fig. 7c), with important physiological interests. For example in the case in lymphocytes which are reservoir cells for MMTV, when reunited, two different stresses: nervous (cortisol, whose action is mediated by GR) and infectious (mediated by Oct-2) (Bendall et al., 1997) can trigger MMTV expression. This can explain how dormant viruses are revived upon a conjunction of stresses. Given that glucocorticoids can lead to the death of lymphocytes, this mechanism allows MMTV to escape the cells before sinking with them. As illustrated in Fig. 7c, MMTV expression can be unambiguously engaged upon acute stresses, while the very low initial slope of the $3 \mathrm{D}$ curve forbids MMTV induction by moderate stresses and buffers stochastic bursts of these TFs. This is a typical example of discernment of gene expression, analogous to a crisis meeting to decide when and how the MMTV should be expressed to evade the host cell when its viability is compromised. There is a long-standing and futile debate to determine if viruses and dehydrated organisms can be considered as living structures or not. By itself, the biochemical discernment described here is sufficient to classify them in the living matter.

\subsection{The zero-order system arises from substrate recycling through two inverted one-way doors}

An outstanding aspect of biochemical systems is that many molecules are continuously modified and de-modified by couples of enzymes with opposite activities. One can cite among others, phosphorylation and dephosphorylation, ubiquitination and de-ubiquitination, sumoylation and desumoylation, methylation and demethylation etc. Since one molecular form only is active (the modified or the de-modified one, depending on the cases), this system has long been perceived as an "interrupter mechanism" determining the activity of the target molecule in an on/off manner. The problem is that the modifying and de-modifying enzymes are present simultaneously in the cell and that their ratio varies in very limited proportions, as measured in immunoblotting experiments. These moderate variations are at first glance deceptive because they don't appear appropriate for regulating in an all-or-not fashion the activity of their target substrates. An explanation of this apparent paradox has been provided in 1981 by (Goldbeter and Koshland, 1981), in a mechanism called zero-order because it is sharper when the enzymes are nearly saturated. This system is an indirect mode of cooperativity since the product of one enzymatic reaction is a substrate for the other one.

In this mechanism, the modifications are macroscopically reversible but microscopically irreversible, so that their steady state ratio can brutally overbalance in case of a slight change in the ratio of the modifying and de-modifying enzymes. Moreover, the sharp sigmoidal dependence of target modification to the linear variation of one modification enzyme, can be obtained with hyperbolic fractional rates. If a substrate is modified by an enzyme with a maximal rate $\alpha$ and a Michaelis constant $K_{\alpha}$ (adimensioned Michaelis constant corresponding to $K_{M}$ / [Substrate]), and then de-modified by an enzyme with maximal rate $\beta$ 
and an adimensioned Michaelis constant $K_{\beta}$, the steady state fraction of modified substrate $x$ results from the balance between two Michaelis-Menten reactions:

$\frac{\alpha(1-x)}{K_{\alpha}+1-x}=\frac{\beta x}{K_{\beta}+x}$

from which

$x=\frac{\beta-\alpha+\alpha K_{\beta}+\beta K_{\alpha}-\sqrt{\left(\beta-\alpha+\alpha K_{\beta}+\beta K_{\alpha}\right)^{2}-4 \alpha K_{\beta}(\beta-\alpha)}}{2(\beta-\alpha)}$

The evolution of $x$ as a function of the activity of one enzyme ( $\alpha$ or $\beta$ ) while keeping constant the other one, gives, following Eq. 4.5.2, a sigmoidal curve (Fig. 8). If further approaching saturation of the enzymes by decreasing their Michaelis constants, the switch becomes very abrupt, similar to a stair step. As shown in Fig. 8, when increasing the activity of only one of the two enzymes, the transition between $\mathrm{S}_{0}$ and $\mathrm{S}_{\mathrm{m}}$ is not linear but sigmoidal and corresponds to the steady state values obtained by intersecting two opposite hyperbolas. The sigmoid in the bottom panel of Fig. 8 is obtained for moderately saturated enzymes, but its steepness is predicted to strongly increase when decreasing the Michaelis constants, to become an instantaneous transition. In this respect, the zero-order switch has been compared to the abrupt phenomenon of phase transition (Koshland, 1987; Berg et al., 2000). Indeed, at atmospheric pressure, very few molecules from one liter of water become gaseous between $5^{\circ} \mathrm{C}$ and $95^{\circ} \mathrm{C}$ while the vast majority of molecules change when increasing the temperature from $99.9999^{\circ} \mathrm{C}$ to $100.0001^{\circ} \mathrm{C}$, which is a typical emerging cooperative phenomenon (Lebowitz, 1999) resulting from the intersection between the antagonistic forces of molecule cohesion and separation. The zero-order mechanism has important biological roles, given that cycles of modification/de-modification are generalized to most post-translational modifications. This process contributes to the capacity of biochemical systems to turn on and turn off its activities in an unambiguous manner. It has also been identified as responsible for the formation of a morphogen gradient in the Drosophila embryo, generated by a smooth gradient of the protein kinase MAPK (Melen et al., 2005). Since different mechanisms are capable to explain the formation of sharp embryonic boundaries, these authors identified the zero-order mechanism based on the resistance of the boundary to substrate concentration changes (Melen et al., 2005).

This mechanism resulting from two antagonistic Michaelis-Menten reactions (Eq. 4.5.1), is possible only thanks to the micro-irreversible nature of these transitions. Indeed, this effect would disappear in a detailed balanced relation between the modified and unmodified forms, whose ratio would be $x /(1-x)$. In this respect, the zero-order enzymatic mechanism is strongly dissipative and very expensive for the cell, since in absence of any signal, the substrates are continuously modified and de-modified at the expense of enzymes, ATP or other molecules. This is an additional example of the cost for the cells, to take unambiguous and appropriate decisions. One should however stress that, as pointed in (Berg et al., 2000), the sharpness of the zero-order mechanism observed in biochemical experiments is likely to be less pronounced in the cell, because of the low copy number of certain molecules.

\section{Non-dissipative sigmoidicity}

All the mechanisms listed in the preceding sections are obtained out of equilibrium; but it will be shown in this section that biochemical discernment can also arise in quasi-equilibrium sub- 
systems, once again by decreasing statistical entropy. This possibility, allowed by the refined organization of macromolecules, is clearly illustrated by the phenomenon of allostery or spatial cooperativity.

\subsection{Spatial cooperativity is the equilibrium counterpart of open kinetic cooperativity: the paradigm of hemoglobin oxygenation}

Spatial cooperativity is a wonderful invention of life because once the molecular actors are evolutionary designed, it complies with the condition of microscopic reversibility; it can work in closed systems and is observable in simple binding isotherms. Typical examples of the role of sigmoidal responses obtained by this way, are provided in appendix 10.3. The most notorious case, clearly observed under full equilibrium conditions, is the sigmoidal oxygenation curve of tetrameric hemoglobin $(\mathrm{Hb})$, which is not possible with its monomeric counterpart myoglobin. The entropic basis of multimeric cooperativity is the decrease of the number of intermediate microstates, compared to that expected for random saturation, as pointed for Ising networks in (Keating and Di Cera, 1993). If the $n$ protomers of a multimer bind to a ligand in a random manner, the shape of the resulting equilibrium binding curve is the same as for isolated protomers, with the same entropy per protomer (Fig. 9c). In turn, if the binding status of certain protomers influences the capacity of adjacent ones to bind the ligand, then, the different possible transitions are no longer equiprobable and the equilibrium curve can become sigmoidal. Macromolecule saturation intermediates are experimentally difficult to identify but steric interferences between clustered protomers have been evidenced.

Different models have been proposed, which all lead to microstate reduction. In the KNF model (Koshland et al., 1966), once a protomer is occupied by the ligand, it strongly favours the filling of one adjacent protomer, thus dramatically limiting the probability of the other possible microstates (Fig. 9a). The situation is more complex for the MWC model (Monod et al., 1965), in which two tetramer conformations coexist, named T and R, leading to $2^{5}$ microstates per molecule. But the principle of microstate reduction is retained, since empty $\mathrm{Hb}$ is refractory to ligand binding ( $\mathrm{T}$ state), while when bound by a ligand molecule, it becomes more receptive to additional ones ( $\mathrm{R}$ state, closer to myoglobin). Hence, the representation of the type T tetramer decreases in the course of saturation. The MWC model, which is a conformational enrichment mechanism, is elegant in that it uses a minimal number of parameters and appears well suited to explain the mode of action of modulators called heterotropic allosteric activators or inhibitors (different from the classical competitive and non-competitive michaelian inhibitors), which can lock the multimer in a given conformation, $\mathrm{R}$ or T respectively (Monod et al., 1965). The MWC model appears remarkably appropriate to the refined regulation of multimeric enzymes such as aspartate transcarbamylase (appendix 10.3).

Although it is sometimes called symmetric, the MWC model is clearly non-symmetric in that only the $\mathrm{R}$ conformation binds efficiently to the ligand. The symmetry of the model resides in the fact that all the subunits are in the same state at any one time and oscillate altogether between the $\mathrm{R}$ and $\mathrm{T}$ states. Hence, such a model would be more compatible with an $\mathrm{Hb}$ made of a single type of protomer, but this is not the case. In this respect, one may wonder why two types of subunits ( $\alpha$ and $\beta$ ), have been selected in human $\mathrm{Hb}$. In fact, this heterogeneous composition suggests an other mode of microstate elimination, resulting from steric hindrance: the hierarchical equilibration mechanism (Michel, 2008). This missed mechanism was in fact implied by the structural studies of Perutz (Perutz, 1970), who showed that when empty, the alpha subunits forbid ligand accessibility to the beta subunits (Fig. 9b). This phenomenon was further supported by nuclear magnetic resonance binding studies performed in equilibrium conditions (Simonneaux et al., 1988). The hierarchical equilibration 
mechanism is as convenient as the MWC model to explain the observation of two conformational states of human $\mathrm{Hb}$, but in addition, it explains its heterogeneous composition. In all the models, the oxygenation curves of $\mathrm{Hb}$ are sigmoidal and their precise equations are available in the corresponding articles (Monod et al., 1965; Koshland et al., 1966; Michel, 2008). The physiological importance of $\mathrm{Hb}$ allostery in the transport of oxygen from the lungs to the other organs is reminded in appendix 10.3. The common feature of all these models is that the elimination of certain saturation intermediates is sufficient to generate sigmoidal fractional saturation curves. This can be also achieved through sequential binding when there is no possibility of choice between binding sites for the ligand at every binding step (Weiss, 1997). The simple elimination of the statistical balancing between micro and macroscopic equilibrium constants, strongly reduces the number of accessible microstates and generates apparent cooperativity with a Hill coefficient of $(n+2) / 3$ where $n$ is the number of clustered binding sites (Michel, 2007).

Interestingly, even if equilibrium cooperativity works without need for cycles and net fluxes as in the previous cases of dissipative cooperativity, the principle of the one-way door, still holds. As schematized in Fig. 9d in the MWC model, an empty subunit R cannot directly recover its conformation $\mathrm{T}$, once a neighbour subunit is liganded. This can be functionally assimilated to a closed door preventing a transition otherwise possible. The existence of a one-way door is even more obvious for the hierarchical equilibration mechanism (Michel, 2008)(Fig. 9e). In this case, the very physical nature of this door has been identified at the molecular level, as reported by Perutz: "In the beta subunits, a side chain of the amino acid valine next to the distal histidine blocked the site that oxygen would have to occupy. The alpha subunits, however, showed no such obstruction" (Perutz, 1978).

Multimeric equilibrium cooperativity has been discovered before monomeric dynamic cooperativity (such as the previous example of glucokinase) and has become a central piece of biochemistry courses, so that this mode of cooperativity now seems familiar. But on reflexion it is in fact the most intriguing one because it allows to acquire a refined biochemical discernment without net fluxes and without heat dissipation. Let us consider the case of $\mathrm{Hb}$ : at equilibrium, $\mathrm{Hb}$ autonomously "decides" to bind less oxygen than it can, when oxygen concentration is lower than a certain threshold. This filtering capacity strikingly resembles that of an informed Maxwell demon, if assimilating the energy of particles to ligand concentration. Accordingly, this phenomenon is clearly associated to a decrease of $H$, as measured by enumerating the microstates (Fig. 9c) and discussed in section 5.4. Equilibrium cooperativity is not restricted to the classical allosteric proteins, but is also encountered in many other aspects of cellular biology.

\subsection{Competitive equilibrium can generate sigmoidicity}

In the zero-order mechanism (section 4.5), if a given amount of substrate is modified and demodified by a couple of competing enzymes, then, the cellular activity governed by one form of the substrate (either modified or de-modified) will be non-linearly dependent on the ratio of the two enzymes. It is interesting to notice that ultrasensitive responses can also be obtained at equilibrium through competitive binding. A typical example is provided by the gene response to a TF subject to sequestration by a poison partner (Buchler and Cross, 2009). If a TF can bind to and activate a gene as a monomer (called $x$ ), which can be inactivated by sequestration into a complex $C$ by a poison partner $(y)$ with a dissociation constant $\mathrm{K}$; and if the total concentrations of the TF and the poison partner are called $X$ and $Y$ respectively, then, the concentrations of the different molecular species obey to the following set of algebraic equations: 
$X=C+x$

$Y=C+y$

$K C=x y$

so that $C$ is the solution of a quadratic equation, as for the zero-order mechanism

$K C=(X-C)(Y-C)$

In these conditions, the saturable fractional gene expression $F$ governed by $x: F=x /\left(K_{D N A}+x\right)$ where $K_{D N A}$ is the dissociation constant between $x$ and DNA, is a strongly sigmoidal function of the $X / Y$ ratio. This behaviour gives the possibility for the cell to take sharp decisions from limited variations of signal inputs.

\subsection{Generalized biases in microstates representation generated by evolutionary-designed macromolecules}

If the mathematical tools of Archibald Hill, such as the Hill coefficient and the Hill plots, remain useful and widely used, it is clear that his aggregation theory to explain the cooperativity of $\mathrm{Hb}$ oxygenation, was wrong and based on the erroneous postulate that independent ligands bind at once $\mathrm{Hb}$. Cooperativity between disconnected molecules would be puzzling and has been more realistically replaced by cooperativity between the connected subunits of $\mathrm{Hb}$ (previous section). But interestingly, the mutual agreement between ligand molecules for binding altogether or none a macromolecule, does exist if two conditions are fulfilled: (i) if the ligand molecules physically interact each other in solution and (ii) if the ligand molecules cannot bind individually to the macromolecule. This is exactly the case for the binding of TF dimers to bipartite DNA elements, for which a genuine Hill equation is uncovered $Y=x^{2} /\left(K+x^{2}\right)$, where $x$ is the concentration of the TF monomer and $K$ is the product of two dissociation constants (that of TF dimerisation and that of binding to DNA of the preformed dimer). A Hill equation with an exponent of 3 can be obtained if the TF binds to DNA as an homo-trimer, such as HSF1. Other modes of cooperative DNA occupation by TFs, which are the very basis of the discernment of gene expression, are listed in (Michel, 2010). In all these cases, the sigmoidicity of the biological response is the result of a reduction of the number of microstates. This is obvious in the case of a TF dimer since the intermediate DNA binding states with a single monomer are inexistent.

\subsection{Equilibrium cooperativity and the second law}

In theory, a system capable of decreasing entropy without need for free energy (as in Fig. 9c, section 5.1), is called a second law-breaking machine. Given that when using probabilistic entropy, the second law seems to be broken by equilibrium cooperativity, it is tempting to deduce that the good definition of entropy is the classical one. But the problem is that the probabilistic view is often very successful, considering for example its singular capacity to simply rule out the Gibbs paradox without denying classical particle individuality. We are aware that decreasing entropy in equilibrium conditions is a Graal of thermodynamics, considered as heretic, following the opinion of Sir Arthur Eddington "If your theory is found to be against the second law of thermodynamics I can give you no hope; there is nothing for it but to collapse in deepest humiliation" (Eddington, 1930). Hence in an attempt to avoid humiliation, let us immediately precise that the second law of thermodynamics is not challenged but refined by incorporating information as an additional component of a 
generalized Carnot principle (section 5.5). In addition, none of the mechanisms listed above can extract work by itself. Eddington was talking about the classical thermodynamic second law, that of heat machines and of the Carnot cycle, which forbids perpetual motion and genesis of work at one temperature only. Even if the filtering action of $\mathrm{Hb}$ results from an equilibrium mechanism, it should be completed by one-way transitions (the diffusion of oxygen in a gradient from blood to tissues) to be converted into physiological activities. Hence, the sigmoidal oxygenation curve of $\mathrm{Hb}$ contributes only indirectly to the possibility for the organism to produce work. For ATCase, the one-way steps of catalysis and/or product release are necessary to score the discernment of ATCase with respect to its substrate concentration (section 10.3). For the equilibrium cooperativity of TFs (section 5.3), the sigmoidal response to dimeric TFs is concretized only if transcription does start, once again through irreversible reactions. But it remains that equilibrium cooperativity could be a fundamental invention of life, whose contribution to cellular discernment and organization is not associated to heat dissipation.

\subsection{Information has a cost}

The different examples selected in section 5 show that many cooperative phenomena in biology can be described using only equilibrium constants. All the dissipating mechanisms described in section 4 can have their equilibrium counterparts. Multimeric allostery is the equilibrium version of dynamic cooperativity of glucokinase (section 4.3). Competitive dimerisation described in section 5.2, is a microreversible reciprocal sequestration mechanism which can work in a manner similar to the zero-order mechanism (section 4.5). The dissipative mechanism of substrate quality control (section 4.1) can sometimes be replaced by finely adjusted evolutionary pre-organized macromolecules with internal cooperativity, but this is not always possible, as in the case of polymerases which should remain capable of accommodating different substrate molecules. These cooperative phenomena rely on refined pre-organization and co-evolution, which result from the possibility to archive moderately successful attempts and to progressively optimize them across generations. Increasing the degree of genome-archived preorganization could decrease the need for dissipative mechanisms. This general principle is not restricted to biological molecules and is also recognized at the level of society, for which information is expected to lower heat dissipation, with ecological benefits. As sensed by Brillouin, entropy $(S)$ and information $(I)$ cannot be examined separately. They are linked in a generalized Carnot principle $\Delta(S-I) \geq 0$, making possible that information can decrease entropy or increase negentropy. In fact, if cumulating the time spent by living structures in the evolution workshop, then, the second principle questioned in section 5.4, is restored. Information has been collected and stored in the genomes at the expense of plenty heat dissipation, which explains the negentropic behaviour of evolved molecules and organisms. The negentropy accumulated in $\mathrm{Hb}$ indirectly provides itself (and cooperatively all the other proteins), with the opportunity to acquire more information by keeping the species alive, in a positive loop maintained across generations. Acquiring information requires negentropy and information can increase negentropy, according to the cycle drawn by Brillouin: Negentropy $\rightarrow$ Information $\rightarrow$ Negentropy (Brillouin, 1956). Archiving 1 bit of information requires at least $k_{B} \ln 2(\mathrm{~J} / \mathrm{K})$, but in the blind Darwinian evolution, this energetic cost is likely to largely exceed this value. Hence, genome evolution can be considered as an expensive investment to decrease the thermodynamic cost of discernment in the future. 


\section{The maintenance of a certain degree of randomness is indispensable for living systems}

Although this review is focused on the conversion of randomness into oriented decisions, it should be reminded that a certain level of uncertainty is maintained in many aspects of cellular biology. For example in metazoans, the cells would not differentiate into specialized phenotypes in absence of arbitrary splitting events. This explains the remarkable cell-to-cell heterogeneity of pluripotent progenitor stem cells, which allows that cell differentiation can proceed more by selection than by induction mechanisms. In unicellular organisms, cellular indecision is also useful for diversifying the phenotypes in the population, thus pre-adapting some individuals to possible conditions changes. These non-discerning splitting events can be obtained by exploiting the natural stochasticity of molecular collisions, for example through decreasing the number of molecules involved in dictating cell fates and by slowering their dynamics. Considerable attention has recently been paid to biological randomness and stochasticity, in many areas of cellular biology and gene expression, so that life is now often considered to be dictated by chance in single cells. However, if stochasticity ensures important biological roles such as for genetic circuits (Raj and van Oudenaarden, 2008; Eldar and Elowitz, 2010), one should keep in mind that the genuine specificity of life is at the exact opposite of this view, particularly in metazoans. Randomness can be easily obtained since it is inherent to small chemical systems, whereas discernment is much more demanding, but is essential for the coherence and longevity of metazoan tissues. The present review deals with mechanisms in steady states, which are the asymptotes of average evolutions; but in addition, specific mechanisms are also involved in the stability, or resistance to fluctuations, of these steady states, in cellular systems made of few molecules or involving few events (Raj and van Oudenaarden, 2008). One can cite for example the reproducible retinal responses to single photons obtained by averaging successive Poisson events (Doan, 2006). Basic means to limit stochastic fluctuations are to increase the number of molecular actors such as enzymes or, for low copy number macromolecules, to increase the frequency of their interactions. The most obvious case of low copy number macromolecule subject to this latter mechanism is the gene (Michel, 2009). As the non-equilibrium steady state mechanisms listed in the study, most noise-reduction mechanisms are also energy-consuming. Failures in cellular discernment, possibly related to a decline of available energy, lead to erratic behaviours typically observed during ageing (Goldstein and Shmookler Reis, 1984; Bahar et al., 2006) and in cancer (Brock et al., 2009). What is good for stem cells is bad for cancer, by allowing fractions of the tumoral population to resist natural or medical anti-cancer defenses. Although their heterogeneity is classically attributed to mutations, it could primarily result from a loss of cellular discernment, as suggested by the existence of mutation-free aggressive cancer cells, which secondarily leads to mutations resulting from repair defects.

\section{How biochemical discernment can arise from the inanimate world}

The emergence of discerning actions from random motions, for which several statistical mechanisms are listed in this study, can be assimilated to the fundament of life, distinguishing it from chemical soups. Of course, the precise ingredients of the primitive soup are unknown but the founder principles can still be recognized in the entropic behaviour of present macromolecules, raising the question of their origin.

\subsection{Organized environment: a condition for life to exist}

The entropic behaviour of life pointed by Boltzmann is not unique to life. It is for example encountered in crystallization and is necessary in the environments capable of incubating life. 
Indeed, life cannot appear anywhere. It requires an anisotropic surrounding and is favoured in a "geologically living" planet. Once appeared on earth, life further amplified chemical gradients and anisotropic distributions, but disequilibria necessarily preexisted before life. The small-scale structure of the observable universe into stars and planets with asymmetrical energy fluxes, also reflects a strong degree of organization. Net fluxes on our planet, such as the flying clouds and rustling torrents, would disappear in absence of sunlight. Chemosynthetic life would have been precluded in an homogeneous environment, and photosynthetic life would have of course been forbidden in absence of light. The origin of this organized environment is a fundamental point, but the question of the entropy of an expanding universe is not trivial since the universe does not behave as an ideal gas system for which a reversible isothermal volume increase would lead to an increase of configurational entropy following $\Delta S=\mathrm{R} \ln \left(V_{2} / V_{1}\right)$. For the universe, the combination of expansion and gravity makes possible that entropy, though increasing, remains farther and farther from its equilibrium value, so that the universe's components can self-organize (Frautschi, 1982).

\subsection{Self-organization out of equilibrium}

Cooperative phenomena are generated by driven biochemical cycles, such as those considered above (section 4). These cycles can be envisioned are elaborated versions of a general principle, not specific of life, allowing, in non-equilibrium conditions, the emergence of organized structures from random individual motions (Lebowitz, 1999). This principle can be readily visualized in very elementary systems such as that noticed by Schrödinger (Schrödinger, 1944): the regular coloured flow collectively generated by the random spreading of individual permanganate molecules in water, when starting from an initial state far from equilibrium. Organized structures can be stationary and evolvable in sustained nonequilibrium conditions. They emerge from symmetry-breaking instabilities and are subject to brutal shifts upon perturbations, as illustrated by fluidic convection cells generated in large temperature gradients (Prigogine, 1977). While the unavoidable microscopic irregularities are immediately cancelled at equilibrium, conversely far from equilibrium, they are explosively amplified, leading the system to split into macroscopic structures. The concept of selforganization has been used for these non-living rudimentary structures, but they are maintained only upon strong exogenous forces, contrary to life which self-controls its own energy refuelling, in a sort of feedback loop. Life is both self-organized and self-maintained away from equilibrium. Finally, we would greatly appreciate being something more than dissipative structures which appear as a somewhat restrictive view of life.

\subsection{Is life a link among others in the chain of matter upgrading phenomena?}

From an astrophysical perspective, the organizational strategies of biochemistry belong to a long chain of matter complexification events initiated in the earlier stages of universe evolution (section 7.1). The formation of galaxies, stars, non-hydrogen atoms, molecules and planets, initiated a chain of organizational mechanisms leading to the onset of biochemical discernment, which is itself an indispensable basis for the emergence of higher order networks. The chain continues with gene networks (whose dissipative nature, with molecules births and decays, is necessary to sculpt network attractors), multi-cellular organisms, neuronal networks (Hopfield, 1982), multi-organismic societies, networking through oral and written language, perhaps indefinitely (Frautschi, 1982). The force underlying this irresistible evolution towards structures exporting more and more entropy in the surrounding (until now), could result from the principle of maximal entropy production in non-equilibrium systems, related to the least action principle. The different theories on the subject go beyond the scope 
of this review, but it would be singular that while the maximal homogenization is predicted in closed systems, reciprocally the maximal organization possible could be expected in perpetuated non-equilibrium conditions. From these considerations, one can envision life as a critical milestone in the self-organization propensity of matter.

\section{Ratchet mechanisms shortening the life's emergence time}

One may wonder if the evolution of matter after the big bang was doomed to generate life, or in other words, if life was already inscribed in the most elementary matter components. Knowing that life appeared on earth, the answer to this question is undoubtedly yes. From a chemical perspective, if life proved possible once, it is just possible; it is a question of time and of windows of opportunity. The micro-reversible appearance of life (state $n$ ) from a chemical soup (state 0) from a chain of $n$ events (Fig. 10) would take an astonishing time because of the very low probability of certain forward transitions. Moreover, every intermediate state can reverse or accidentally dissolve in the environment (irreversible side reactions of rate $k^{0}$ in Fig. 10), thus aborting the chain. In addition, the rates of certain backward transitions $\left(k^{-}\right)$are likely to largely exceed the forward ones $\left(k^{+}\right)$. The overall forward waiting time to complete the whole chain when starting from an untransformed starting state 0 (Fig. 10), is

$T_{0 \rightarrow n}=\sum_{i=1}^{n} \prod_{j=1}^{i} \frac{k_{j-1}^{-}+k_{j-1}^{0}}{k_{j}^{+}}$

by setting $\Sigma k_{0}=1$ (unitless). This time is expected to be very long but the presence of some one-way arrows in the chain, corresponding to critical jumps in the self-organizing evolution of matter, can strongly shorten the delay through a ratchet mechanism. For example, if introducing a one-way door between the $(q-1)^{\text {th }}$ and the $q^{\text {th }}$ states in the chain of Fig. 10, the delay reduces to:

$T_{0 \rightarrow n}=\sum_{i=1}^{q} \prod_{j=1}^{i} \frac{k_{j-1}^{-}+k_{j-1}^{0}}{k_{j}^{+}}+\sum_{i=q+1}^{n} \prod_{j=q+1}^{i} \frac{k_{j-1}^{-}+k_{j-1}^{0}}{k_{j}^{+}}$

and so on. Writing Eq. 8.2 is longer, but T is shorter. Once the $q^{\text {th }}$ step is reached, there is no need, as in the myth of Sisyphus, to restart again and again the $q$ - 1 first steps of the chain. These one-way transitions can be provided by the system itself such as, over a certain threshold of complexity:

(i) Compartmentalization, which allows to control the chemical composition and molecule concentrations at a level compatible with the mechanisms listed in this article, by adjusting them to the sensitivity ranges. Membrane barriers also permit the building of barrages to accumulate potential energy and become less dependent on the uncertain free energy provision from the surrounding soup. The evolutionary onset of proton-motive forces is analyzed in (Lane et al., 2010).

(ii) The capacity to control the intake, conversion and delivery of environmental matter and energy, necessary to keep the system away from equilibrium.

(iii) The capacity to numerically archive the successful recipes on an inert support, relatively resistant to moderate physical and chemical conditions and easily duplicatable and reparable (such as DNA). This fundamental step is a turning point for the rapid improvement 
of living systems, by allowing the replacement of dynamic entropy by pure information. The advent of genomes has primed the amplification loop between negentropy and information described in section 5.5, leading to a powerful acceleration of complexity increase. This tendency could progressively alleviate the dissipation of entropy associated to life complexification. The outbreak of self-replication of transmissible information supports is generally assimilated to the origin of life, considered as a product of "replicative chemistry" (Pross, 2003). Replicable information molecules can be early and essential, but not initial, milestones of life. This potentiality conferred a decisive advantage to the first form which found it, by allowing the self-maintenance of a given state. Once the negentropy-information cycle of Brillouin is primed, the mechanisms of life and evolution become clear but the fundamental unsolved question is the initial discernment allowing its priming. An additional explanation of the common origin of present living structures on earth, complementary to the arbitrariness of the genetic code, could be that the first heterotrophic self-replicating structure, has eaten the primitive soup and cleared it from organic macromolecules, thus precluding the possibility of development of concurrent forms of life.

The evolution of elaborated forms of life can no longer be modeled on the stochastic basis of Eqs. 8. Questions have long been raised about the true randomness of mutations, in relation with the question of the genetic assimilation of (Waddington, 1959) and in line with the recent evidences of non-random mechanisms in evolutionary processes, including gene network-based differential sensitivity to mutations (Crombach and Hogeweg, 2008), nonrandom meiotic recombination (Khil and Camerini-Otero, 2009) and trans-generational inheritance of epigenetic marks, observed in mammals (Carone et al., 2010) as well as in plants (Hauser et al., 2011). Hence, life could be to some extent, able to direct its own evolution following a so-called Lamarkian view (Daxinger and Whitelaw, 2010), corresponding in fact to the intuitive feeling of both Darwin and Lamarck, in absence of any knowledge of the underlying molecular mechanisms (Noble, 2010). To prolong this view, a major ratchet step, equivalent to a one-way arrow in the chain of Fig. 10, could appear when life will intentionally design secondary forms of self-improving intelligence and life.

\section{Discussion}

A series of elementary processes is listed in section 4, which provide altogether a concrete illustration of the idea of Ilya Prigogine that matter "begins to see" out of equilibrium. In a large network at equilibrium (say made of thousands of components), as noticed by Lewis (Lewis, 1925), between any two connected nodes, the same number of transitions is expected over time in both directions. But strikingly, introducing a single one-way arrow in the network is sufficient to break micro-reversibility everywhere in the network. This common basis of the dissipative mechanisms listed here, concretely illustrates a mode of injection of the external inputs long postulated to maintain life away from equilibrium. These mechanisms concur to filter randomness by amplifying slight differences of binding potential or of substrate concentration and by functionally eliminating noisy and irrelevant molecules. Cooperative phenomena also allow the discerning management of high-dimensional networks, by limiting the number of actors and connections necessary for the differential regulation of a huge number of elements. Microstate reduction in molecular interactions leads to non-linear relations in regulatory networks. In turn, non-linear functions are basic ingredients for the capacity of gene networks to generate patterning, multistability and attractors, which correspond in metazoans to cell type-specific differentiation states (Jacob and Monod, 1963; Kauffman, 2004). 
A strong evolutionary acceleration is rendered possible by the setting up of transmissible information-archiving devices. This step has primed the wonderful positive feedback between negentropy increase and information acquisition and storage. It opened a new era of selforganization in which certain roles of time-irreversible transitions ensured by moderately preorganized macromolecules, can be transferred to time-reversible cooperative phenomena governed by finely preorganized macromolecules. Examples of such processes are presented in section 5. This evolution, associated to a decrease of heat dissipation, corresponds to a change in the nature of life entropy, shifting from dynamical to informational, while remaining basically statistical. This evolution could recapitulate the correspondence pointed by Brillouin between thermodynamic and information entropy, which are two facets of the same principle (Brillouin, 1956). The Boltzmann's entropy derived from statistical mechanics is only one aspect of entropy, in which asymmetry is expected only out of equilibrium. In fact, asymmetric behaviours can emerge in a microreversible manner from highly preorganized macromolecules, such as the remarkable MWC enzymes, which can coordinate several biosynthetic pathways in the cell by heterotropic regulation (the example of ATCase is summarized in appendix 10.3). The shift of entropy from statistical dynamics to information, can be transposed to the higher organisational levels of the society's education.

The organizational roles of proteins involved in the cooperative phenomena listed here, depend on their critical ability to introduce biases between possible reactions, thanks to their flexible, elastic and plastic nature. This is the case for the slow relaxation of proteins generating molecular memory effects and for proteins expanding their binding sites to test the strength of ligand association. These properties can appear only in macromolecules since increasing the number of atoms and bonds in molecules, increases the number of possible metastable conformations. It is worth noting that the tridimensional shape of proteins, is itself an energy-dependent epigenetic phenomenon. Most proteins would be disordered without the assistance of protein chaperones. In this respect, those capable to find spontaneously their active conformation and called autochaperones, such as RNase A or ubiquitin, are the exception to the rule. Certain folding chaperones have ATPase domains and help their protein substrates to acquire their proper conformation through reiterative, strongly energyconsuming, vibrating cycles of partial unfolding and refolding, which is a typical example of energy-dependent elimination of undesirable states.

Symmetry has long been used as a criterion of an elegant physical law, and its quest inspired the conception of fundamental models, but asymmetries clearly exist and propagate in the universe. Life emerged from these preexisting asymmetries and strikingly concentrated them. From the generalized chiral imbalance of its components to the asymmetric fluxes and microstate reduction, life appears as a form a rebellion against symmetry and uniformization, directly related to the struggle for entropy sensed by Ludwig Boltzmann (Boltzmann, 1886). Finally, it is intriguing to notice how our world appears as a succession of layers of emergence. The collective discerning behaviours listed in the present review emerge from randomly moving distinguishable particles, which themselves are emergent properties of the quantum underworld. In this chain of complexification, life could be an essential link, for its capacity to introduce the management of information in the future modes of cooperativity and of matter organization. 


\section{Appendices}

10.1. Comparative rate of fixation of two ligands in the simple Michaelis-Menten reaction.

To evaluate the competition between correct $(S)$ and wrong $\left(S^{\prime}\right)$ substrates in the HopfieldNinio mechanism (Fig. 5b), their rates of productive initial binding (i.e. leading to their effective fixation, written $k_{T}$ in the following equations), are compared in the MichaelisMenten reaction (Fig. 5a). As long as the unique active site of the enzyme is occupied by a given substrate, no other one can access it. The rate of substrate incorporation into $P$ is

$k_{T}=\frac{k_{c} k_{a}[S]}{k_{d}+k_{c}}$

Hence

$R=\frac{k_{T\left(S^{\prime}\right)}}{k_{T(S)}}=\frac{k_{a}^{\prime}\left(k_{d}+k_{c}\right)}{k_{a}\left(k_{d}^{\prime}+k_{c}\right)}$

If the enzyme works too rapidly and immediately ligates the substrate once it arrives $\left(k_{c}>>k_{d}\right.$, $k_{d}^{\prime}$ ), one obtains

$R=\frac{k_{a}^{\prime}}{k_{a}}$

that is to say a complete absence of discrimination between wrong and correct substrates (The first arrived wins). If the binding rates of the resembling substrates are identical $\left(k_{a}=k_{a}^{\prime}\right)$, we have

$R=\frac{k_{d}+k_{c}}{k_{d}^{\prime}+k_{c}}$

and if there is a time scale separation between the binding and the catalytic reactions

$R=\frac{k_{d}}{k_{d}^{\prime}}$

10.2. The Wegscheider relation.

Fig $6 \mathrm{C}$ represents the mixed induced fit/conformational selection cycle. The saturation curve obtained using steady state methods and including all the reaction rates, is complex, useless and contains square concentrations of the ligand (not shown). But the eight rate constants of this cycle cannot be arbitrarily chosen because they are mutually constrained by the Wegscheider relation, which strikingly simplifies the equation and eliminates the square concentrations. The forward/reverse rate ratios depend on the relative energies of the connected states, according to:

$\frac{k_{a}[L]}{k_{d}}=\mathrm{e}^{\left(E_{M}-E_{M L}\right)}$
$\frac{k_{i}}{k_{-i}}=\mathrm{e}^{\left(E_{M L}-E_{M^{\prime} L}\right)}$ 
$\frac{k_{d}^{\prime}}{k_{a}^{\prime}[L]}=\mathrm{e}^{\left(E_{M^{\prime} L}-E_{M^{\prime}}\right)}$

$\frac{k_{r}}{k_{-r}}=\mathrm{e}^{\left(E_{M^{\prime}}-E_{M}\right)}$

where $\mathrm{E}$ is the dimensionless normalized particle energy. By compressing Eq. 10.2, one obtains:

$\frac{k_{a}[L] k_{i} k_{d}^{\prime} k_{r}}{k_{d} k_{-i} k_{a}^{\prime}[L] k_{-r}}=\mathrm{e}^{\left(E_{M}-E_{M L}+E_{M L}-E_{M^{\prime} L}+E_{M^{\prime} L}-E_{M^{\prime}}+E_{M^{\prime}}-E_{M}\right)}=1$

Hence

$k_{r} k_{a} k_{i} k_{d}^{\prime}=k_{-r} k_{a}^{\prime} k_{-i} k_{d}$

10. 3. Typical outcomes of time-reversible cooperativity.

Two cases of micro-reversible discerning biochemical activities are illustrated in Fig. 11.

a) The enzyme aspartate transcarbamylase (ATCase) from E. coli is a good example of discerning allosteric enzyme. ATCase catalyses the first reaction of a chain leading to the synthesis of pyrimidines from aspartate. Pyrimidines are components of the pyrimidic nucleotides of nucleic acids ( $\mathrm{C}$ and $\mathrm{T}$ in DNA). They should be present in the same amount than purine nucleotides ( $G$ and $A$ ) in the cell, because they enter in roughly equivalent proportions into the composition of nucleic acids, according to Watson-Crick base pairing. Since the synthetic pathway of purines is chemically disconnected from that of pyridines, ATCase has evolved to adapt its activity to the purine/pyrimidine ratio. The ATP and CTP molecules act as "allosteric modulators" of the ATCase activity, to continuously adjust the synthesis of pyrimidines to the amount of purines. ATCase is a dimeric protein and the MWC model of allostery is particularly well suited to explain this behaviour (Monod et al., 1965).

b) Saturation curve of $\mathrm{Hb}$ (sigmoidal) or myoglobin (hyperbolic) with oxygen in normal coordinates. The main physiological interest of the sigmoidal behavior of $\mathrm{Hb}$ is the large difference of saturation $\left(\Delta Y_{S}\right)$ between lungs and peripheral organs, allowing the greatest part of oxygen loaded in the lungs to be released in the tissues. Intra-muscular myoglobin, which resembles a single subunit of $\mathrm{Hb}$ and has an hyperbolic saturation curve, further helps transferring oxygen from blood to red muscles. The capacity of $\mathrm{Hb}$ to bind as much oxygen as it can in the lungs and to bind as little oxygen as it can in the muscles, is a spontaneous nondissipative discriminating behaviour (section 5).

\section{References}

Archer, T.K., Lefebvre, P., Wolford, R.G., Hager, G.L. 1992 Transcription factor loading on the MMTV promoter: a bimodal mechanism for promoter activation. Science 255, 1573-1576.

Atkinson, D.E. 1977 Cellular energy metabolism and its regulation. Academic Press, New York 
Bahar, R., Hartmann, C.H., Rodriguez, K.A., Denny, A.D., Busuttil, R.A., Dollé, M.E., Calder, R.B., Chisholm, G.B., Pollock, B.H., Klein, C.A., Vijg, J. 2006 Increased cellto-cell variation in gene expression in ageing mouse heart. Nature 441, 1011-1014.

Beard, D.A., Qian, H. 2007 Relationship between thermodynamic driving force and one-way fluxes in reversible processes. PLoS One 2, e144.

Belikov, S., Holmqvist, P.H., Astrand, C., Wrange, O. 2004 Nuclear factor 1 and octamer transcription factor 1 binding preset the chromatin structure of the mouse mammary tumor virus promoter for hormone induction. J Biol Chem 279, 49857-67.

Bendall, H.H., Schrerer, D.C., Edson, C.R., Ballard, D.W., Oltz, E.M. 1997 Transcription factor NF-kB regulates inducible Oct-2 gene expression in precursor B lymphocytes. J. Biol. Chem. 272, 28826-28828.

Berg, O.G., Paulsson, J., Ehrenberg, M. 2000 Fluctuations and quality of control in biological cells: zero-order ultrasensitivity reinvestigated. Biophys J. 79, 1228-1236.

Bintu, L., Buchler, N.E., Garcia, H.G., Gerland, U., Hwa, T., Kondev, J., Phillips, R. 2005 Transcriptional regulation by the numbers: models. Curr. Opin. Genet. Dev. 15, 116124.

Bolouri, H., Davidson, E.H. 2002 Modeling DNA sequence-based cis-regulatory gene networks. Dev. Biol. 246, 2-13.

Boltzmann, L. 1886 Der zweite Hauptsatz der mechanischen Wärmetheorie. Almanach der kaiserlichen Akademie der Wissenschaften 36, 225-259.

Brillouin, L. (1956) Science and information theory, Academic Press, Inc., New York.

Brock, A., Chang, H., Huang, S. 2009 Non-genetic heterogeneity - A mutation-independent driving force for the somatic evolution of tumours. Nat. Rev. Genet. 10, 336-342.

Buchler, N.E., Cross, F.R. 2009 Protein sequestration generates a flexible ultrasensitive response in a genetic network. Mol. Syst. Biol. 5, 272.

Cárdenas, M.L., Rabajille, E., Niemeyer, H. 1979 Kinetic cooperativity of glucokinase with glucose. Arch. Biol. Med. Exp. (Santiago) 12, 571-580.

Carone, B.R., Fauquier, L., Habib, N., Shea, J.M., Hart, C.E., Li, R., Bock, C., Li, C., Gu, H., Zamore, P.D., Meissner, A., Weng, Z., Hofmann, H.A., Friedman, N., Rando, O.J. 2010 Paternally induced transgenerational environmental reprogramming of metabolic gene expression in mammals. Cell 143, 1084-1096.

Chávez, S., Beato, M. 1997 Nucleosome-mediated synergism between transcription factors on the mouse mammary tumor virus promoter. Proc. Natl. Acad. Sci. USA 94, 28852890.

Cherry, J.L., Adler, F.R. 2000 How to make a biological switch. J.Theor. Biol. 203, 117-133.

Cornish-Bowden, A., Cárdenas, M.L. 1987 Co-operativity in monomeric enzymes. J. Theor. Biol. 124, 1-23.

Cornish-Bowden, A., Cárdenas, M.L. 2008 Self-organization at the origin of life. J. Theor. Biol. 252, 411-418.

Crombach, A., Hogeweg, P. 2008 Evolution of evolvability in gene regulatory networks. PLoS Comput. Biol. 4, e1000112.

Daxinger, L., Whitelaw, E. 2010 Transgenerational epigenetic inheritance: more questions than answers. Genome Res. 20, 1623-1628.

Doan, T., Mendez, A., Detwiler, P.B., Chen, J., Rieke, F.(2006) Multiple phosphorylation sites confer reproducibility of the rod's single-photon response. Science313, 530-533.

Eddington, A. (1930) The Nature of the physical world, MacMillian, New York.

Eisfeld, K., Candau, R., Truss, M., Beato, M. 1997 Binding of NF1 to the MMTV promoter in nucleosomes: influence of rotational phasing, translational positioning and histone H1. Nucl. Acids Res. 25, 3733-3742. 
Eldar, A., Elowitz, M.B. 2010 Functional roles for noise in genetic circuits. Nature 467, $167-$ 173.

Frautschi, S. 1982 Entropy in an expanding universe. Science 217, 593-599.

Fryer, C.J., Archer, T.K. 1998 Chromatin remodelling by the glucocorticoid receptor requires the BRG1 complex. Nature 393, 88-91.

Gilbert, W. 1986. Origin of life: the RNA world. Nature 319, 618-628.

Goldbeter, A., Koshland, D.E. 1981 An amplified sensitivity arising from covalent modification in biological systems. Proc. Natl. Acad. Sci. U.S.A.

Goldstein, S., Shmookler Reis, R.J. 1984 Genetic modifications during cellular aging. Mol Cell Biochem. 64, 15-30.

Hammes, G.G., Chang, Y.C., Oas, T.G. 2009 Conformational selection or induced fit: a flux description of reaction mechanism. Proc. Natl. Acad. Sci. USA 106, 13737-13741.

Hauser, M.T., Aufsatz, W., Jonak, C., Luschnig, C. 2011 Transgenerational epigenetic inheritance in plants. Biochim. Biophys. Acta. [Epub ahead of print].

Hebbar, P.B., Archer, T.K. 2007 Chromatin-dependent cooperativity between site-specific transcription factors in vivo. J Biol Chem 282, 8284-91.

Heredia, V.V., Thomson, J., Nettleton, D., Sun, S. 2006 Glucose-induced conformational changes in glucokinase mediate allosteric regulation: transient kinetic analysis. Biochemistry 45, 7553-7562.

Hopfield, J.J. 1974 Kinetic proofreading: a new mechanism for reducing errors in biosynthetic processes requiring high specificity. Proc. Natl. Acad. Sci. USA 71, 4135-4139.

Hopfield, J.J. 1982 Neural networks and physical systems with emergent collective computational abilities. Proc. Natl. Acad. Sci. USA. 79, 2554-2558.

Jacob, F., Monod, J. (1963) Genetic repression, allosteric inhibition, and cellular differentiation. In Cytodifferential and macromolecular synthesis, (ed. M. Locke), pp. 30-64, Acad. Press. Inc., New York.

Jaynes, E.T. 1957 Information theory and statistical mechanics. Physical Review 106, 620630.

Kauffman, S. 2004 A proposal for using the ensemble approach to understand genetic regulatory networks. J. Theor. Biol. 230, 581-590.

Kaufmann, M. 2009 On the free energy that drove primordial anabolism. Int. J. Mol. Sci. 10, 1853-1871.

Keating, S., Di Cera, E. 1993 Transition modes in Ising networks: an approximate theory for macromolecular recognition. Biophys. J. 65, 253-269.

Khil, P.P., Camerini-Otero, R.D. 2009 Variation in patterns of human meiotic recombination. Genome Dyn. 5, 117-127.

Khrapunov, S., Brenowitz, M., Rice, P.A., Catalano, C.E. 2006 Binding then bending: A mechanism for wrapping DNA. Proc. Natl. Acad. Sci. USA 103, 19217-19218.

King, E.L., Altman, C. 1956 A schematic method of deriving the rate laws for enzymecatalyzed reactions. J. Phys. Chem. B. 60, 1375-1378.

Koshland, D.E. 1958 Application of a theory of enzyme specificity to protein synthesis. Proc. Natl. Acad. Sci. U.S.A. 44, 98-104.

Koshland, D.E., Nemethy, G., Filmer, D. 1966 Comparison of experimental binding data and theoretical models in proteins containing subunits. Biochemistry 5, 365-385.

Koshland, D.E. 1987 Switches, thresholds and ultrasensitivity. Trends. Biochem. Sci. 12, 225229.

Lane, N., Allen, J.F., Martin, W. 2010 How did LUCA make a living? Chemiosmosis in the origin of life. Bioessays 32, 271-280.

Lebowitz, J.L. 1993 Boltzmann's Entropy and Time's Arrow. Physics Today 46, 32-38. 
Lebowitz, J.L. 1999 Cooperative behavior in Simple and Complex Systems. Science for Survival and Sustainable Development, Pointifical Academy of Sciences, Scripta Varia, 98 98, 321-325.

Lewis, G.N. 1925 A New Principle of Equilibrium. Proc. Natl. Acad. Sci. USA 11, 179-183.

Lin, S.X., Neet, K.E. 1990 Demonstration of a slow conformational change in liver glucokinase by fluorescence spectroscopy. J. Biol. Chem. 265, 9670-9675.

Masters, K.M., Parkhurst, K.M., Daugherty, M.A., Parkhurst, L.J. 2003 Native human TATAbinding protein simultaneously binds and bends promoter DNA without a slow isomerization step or TFIIB requirement. J. Biol. Chem. 278, 31685-31690.

Melen, G.J., Levy, S., Barkai, N., Shilo, B.Z. 2005 Threshold responses to morphogen gradients by zero-order ultrasensitivity. Mol. Syst. Biol. 1, 0028.

Michel, D. 2007 Cooperative equilibrium curves generated by ordered ligand binding to multi-site molecules. Biophys. Chem. 129, 284-288.

Michel, D. 2008 An alternative theoretical formula for hemoglobin oxygenation. Eur. Biophys. J. 37, 823-827.

Michel D. 2009 Fine tuning gene expression through short DNA-protein binding cycles. Biochimie. 91, 933-941.

Michel, D. 2010 How transcription factors can adjust the gene expression floodgates. Prog. Biophys. Mol. Biol. 32, 16-37.

Michel, D. 2011 Hierarchical cooperativity mediated by chromatin remodeling; the model of the MMTV transcription regulation. J. Theor. Biol. 287, 74-81.

Min, W., Xie, X.S., Bagchi, B. 2009 Role of conformational dynamics in kinetics of an enzymatic cycle in a nonequilibrium steady state. J Chem Phys. 131, 065104.

Mirny, L.A. 2010 Nucleosome-mediated cooperativity between transcription factors. Proc. Natl. Acad. Sci. U.S.A. 107, 22534-22539.

Monod, J., Wyman, J., Changeux, J.-P. 1965 On the nature of allosteric transitions: a plausible model. J. Mol. Biol. 12, 88-118.

Ninio, J. 1975 Kinetic amplification of enzyme discrimination. Biochimie 57, 587-595.

Noble, D. 2010 Biophysics and systems biology. Phil. Trans. R. Soc. A 368, 1125-1139.

Parvin, J.D., McCormick, R.J., Sharp, P.A., Fisher, D.E. 1995 Pre-bending of a promoter sequence enhances affinity for the TATA-binding factor. Nature 373, 724-727.

Perutz, M.F. 1970 Stereochemistry of cooperative effects in haemoglobin. Nature 228, 726739.

Perutz, M.F. 1978 Hemoglobin structure and respiratory transport. Scientific Am. 259, 68-86.

Prigogine, I. 1977 Time, structure and fluctuations. Nobel lecture 8 December, http://nobelprize.org/nobel_prizes/chemistry/laureates/1977/prigogine-lecture.html.

Pross, A. 2003 The driving force for life's emergence: kinetic and thermodynamic considerations. J. Theor. Biol. 220, 393-406.

Rabin, B.R. 1967 Co-operative effects in enzyme catalysis: a possible kinetic model based on substrate-induced conformation isomerization. Biochem J. 102, 22C-23C.

Raj, A., van Oudenaarden, A. 2008 Nature nurture, or chance: stochastic gene expression and its consequences. Cell 135, 216-226.

Schrödinger, E. (1944) What is life - the physical aspect of the living cell, Cambridge University Press, Cambridge.

Segal, E., Widom, J. 2009 From DNA sequence to transcriptional behaviour: a quantitative approach. Nat. Rev. Genet. 10, 443-456.

Simonneaux, G., Bondon, A., Brunel, C., Sodano, P. 1988 Direct observation of intermediate ligation states of hemoglobin. J. Am. Chem. Soc. 110, 7637-7640.

Sugimura, S., Crothers, D.M. 2006 Stepwise binding and bending of DNA by Escherichia coli integration host factor. Proc. Natl. Acad. Sci. USA. 103, 18510-18514. 
Sullivan, S.M., Holyoak, T. 2008 Enzymes with lid-gated active sites must operate by an induced fit mechanism instead of conformational selection. Proc. Natl. Acad. Sci. USA $105,13829-13834$.

Tolman, T.C. 1925 The principle of microscopic reversibility. Proc. Natl. Acad. Sci. U.S.A. $11,436-439$.

Trifonov, E.N. 2009 The origin of the genetic code and of the earliest oligopeptides. Res. Microbiol. 160, 481-486.

Vaneechoutte, M., Fani, R. 2009 From the primordial soup to the latest universal common ancestor. Res. Microbiol. 160, 437-440.

Vichi, P., Coin, F., Renaud, J.P., Vermeulen, W., Hoeijmakers, J.H., Moras, D., Egly, J.M. 1997 Cisplatin- and UV-damaged DNA lure the basal transcription factor TFIID/TBP. EMBO J. 16, 7444-7456.

Waddington, C.H. 1959 Evolutionary systems; animal and human. Nature 183, 1634-1638.

Wang, G.M., Sevick, E.M., Mittag, E., Searles, D.J., Evans, D.J. 2002 Experimental demonstration of violations of the second law of thermodynamics for small systems and short iime scales. Phys. Rev. Lett. 89, 050601.

Weikl, T.R., von Deuster, C. 2009 Selected-fit versus induced-fit protein binding: kinetic differences and mutational analysis. Proteins 75, 104-110.

Weiss, J.N. 1997 The Hill equation revisited: uses and misuses. FASEB J. 11, 835-841.

Whitehead, E. 1970 The regulation of enzyme activity and allosteric transition. Prog. Biophys. Mol. Biol. 21, 321-397.

Wolfe-Simon, F., Blum, J.S., Kulp, T.R., Gordon, G.W., Hoeft, S.E., Pett-Ridge, J., Stolz, J.F., Webb, S.M., Weber, P.K., Davies, P.C., Anbar, A.D., Oremland, R.S. 2010 A bacterium that can grow by using arsenic instead of phosphorus. Science 332, 1163 1166.

Zhao, X., Herr, W. 2002 A regulated two-step mechanism of TBP binding to DNA: a solventexposed surface of TBP inhibits TATA box recognition. Cell 108, 615-627. 


\section{Figures legends}

Figure 1. (a) Box with three identical gates between three space units A, B, C. (b) One-way door, allowing in this scheme the passage of the balls from left to right, but forbidding it in the reverse direction. (c) Insertion, between the compartments $\mathrm{B}$ and $\mathrm{C}$, of a one-way door which opens only for the balls coming from B. (d) Representation of the system in a space transition graph. Given that all the gates have the same width, all the rate constants are identical $(k)$. (e)-(f) These two snapshots are microstates and as such, they have the same probability to occur, but the panel $\mathbf{e}$ is perceived as more typical, based on our intuitive probabilistic feeling. (e)-(g) The same boxes as in the panels a and c, when increasing the number of balls.

Figure 2. The two inputs capable of maintaining one-way transitions in open systems: (a) Energy supply. One pivotal invention of biochemical systems is the molecular adjustments allowing to couple together a reaction strongly favoured thermodynamically with an unfavourable one. (b) Matter provision. The new molecule $\mathrm{C}$ can fall of the binding site of the enzyme and then functionally disappear if its concentration in the system is very low, or if it is immediately incorporated in other complexes or polymerized macromolecules. Such oneway transitions are generalized in the cell, in biosynthetic pathways and in the genetic expression cascade from genes to proteins. (c) Continuous gene product synthesis and degradation is a condition for the structuration of gene networks into attractors. The example shown is a simple positive feedback.

Figure 3. (a) Sigmoidal $v s$ hyperbolic fractional activity curves. The sigmoidal shape presents favourable features for controlling biological responses: (i) the low initial slope allows to filter random signal fluctuations, thus avoiding inappropriate responses and (ii) for smooth sigmoids, the near-linear part of the curve around the inflection point allows to graduate the response within a given range of signal intensity (grayscale bar). In dissipative networks where the concentration of the components result from the balance between production (either non-cooperative or cooperative) and degradation (green straight line; supposed to be not saturable and to follow a first-order rate), the sigmoidal curves, contrary to non-sigmoidal ones, can generate bistability, as illustrated by the number of crossings with the degradation line (green disks). (b) Steeper sigmoids ensure several key aspects of biological discernment: (i) They allow to amplify the discrimination between nearby signals ( $\mathrm{s} 1$ and s2), by pushing away their respective effects (spaced by $\Delta$ s instead of $\Delta h$ ). (ii) They create a threshold effect ensuring that a biological activity is triggered only when the required combination of conditions is reached. (iii) They completely wipe out noisy signals. The pulling force stretching sigmoidal curves, symbolized by the hands, is provided by energy consumption and reflects the thermodynamic cost of decisions and accuracy.

Figure 4. Two consequences of one-way transitions in steady state cycles. (a) Discrimination between substrates based on their dissociation rates. The transitions between the different states of the enzyme E, are represented: (i) pseudo-first order binding to a substrate $\mathrm{S}$ and (ii) transformation into an activated state ES* coupled to an exergonic reaction at a frequency $k^{*}$. The sigmoidal dependence on $k_{d}$ of the reaction rate, reflects the capacity of the system to increase small differences of substrate dissociation rates in a non-linear manner, thus 
preventing undesired reactions. (b) Amplification of differences of substrate concentrations, following the broken induced fit mechanism described in sections 4.2 and 4.3..

Figure 5. The classical Michaelis-Menten reaction (a) is compared to the kinetic substrate selection network (b). The enzyme-substrate complex should be transformed into an activated complex (ES*) through an energy-driven reaction $\left(k^{*}\right)$ for allowing the catalytic reaction $\left(k_{c}\right)$, which offers an additional possibility for the substrate to escape the enzyme.

Figure 6. (a-d) Strong ligand-selection and induced fit mechanisms are mutually exclusive at equilibrium. Pseudo-first order binding equilibria between a ligand L (black triangle) and a macromolecule M. M' corresponds to a conformational variant of M. (a) Pure hyperbolic binding of rigid molecules. (b) Ligand-induced conformational stabilization. (c) Ligandinduced fit $\left(k_{a} \cdot k_{i}\right)$ coupled to conformational selection $\left(k_{-r} \cdot k_{a}{ }^{\prime}\right)$. This complete cycle can be obtained only if the induced fit stabilization is moderate or if tight induced fit is broken by product release. (d) Strong ligand-induced fit, leading to its trapping in a molecular complex. (e-f) Catalytic breaking of the micro-reversible induced fit/conformal selection cycle between an enzyme and its substrate. (e) In absence of catalysis, the binding and conformation cycle should comply with the detailed balance condition, linking together the reaction constants (appendix 10.2) (f) The dissociation of E'S by product formation and escape, allows to break the detailed balance, thus generating a sigmoidal substrate concentration-dependence curve.

Figure 7. Hierarchical DNA-binding cooperativity through chromatin remodeling. (b) Simplified scheme of cooperative TF binding to DNA in equilibrium. Left panel: reciprocal cooperativity. Middle panel: hierarchical cooperativity between physically-interacting TFs. Right panel: hierarchical cooperativity between non-interacting TFs; the scheme illustrates the particular case of DNA allostery. (b) Non-equilibrium hierarchical TF binding, The initial steps of MMTV promoter activation. NF1 and Oct TFs cannot access DNA wrapped around a nucleosome (grey), contrary to GR. Upon corticol treatment, GR binds to the GRE and recruits the SWI/SNF ATPase BRG1, which displaces the nucleosomes and allows NF1 and Oct to bind. (c) Consequences on gene expression of hierarchical cooperativity between two TFs: TF1 opens the ways to TF2, which cannot have an effect alone. The overadditive effect of the combination of TF1+TF2 can be further adjusted by controlling the activity of chromatin remodeling complexes.

Figure 8. The zero-order mechanism of substrate modification/de-modification by $\mathrm{E}_{\mathrm{u}->\mathrm{m}}$ and $\mathrm{E}_{\mathrm{m}->\mathrm{u}}$ michaelian enzymes respectively. When increasing linearly one of these enzymes while keeping constant the other one, the resulting steady state values of the modified substrate $(\mathrm{Sm})$ relatively to the unmodified one $(\mathrm{Su})$, varies abruptly.

Figure 9. Equilibrum cooperativity of allosteric macromolecule saturation. (a) Microstates corresponding to the progressive saturation of a tetrameric macromolecule according to the KNF model in which all the 14 possible saturation intermediates exist, but the probability of certain is increased once the first binding site is occupied, as illustrated by the bold line. (b) In the hierarchical equilibration mechanism, certain transitions are forbidden, thus dramatically reducing the number of intermediate states (to 5). (c) Binding entropy per protomer as a 
function of ligand concentration. Continuous line: for a mixture of monomers with low $(K=1$ concentration $\left.^{-1}\right)$ and high ( 3 -fold higher) affinity $\left(K=3\right.$ concentration $\left.^{-1}\right)$. The same curve is obtained after arrangement of the protomers into tetramers containing two monomers with low affinity and two monomers with high affinity, filled in a random manner. Dotted line: obtained when the same tetramers are saturated in a hierarchical cooperative manner. (d) Hidden one-way doors in the MWC model, drawn for a dimer. The conformational equilibrium between the $\mathrm{T}$ and $\mathrm{R}$ forms involves simultaneously the two protomers because they are mutually constrained. Ligand can bind first to either R1 or R2 with the same probability. As a result, the $\mathrm{R}$ to $\mathrm{T}$ re-conversion is forbidden for the unliganded protomer ( $\mathrm{R} 2$ is arbitrarily chosen in the scheme). (e) One-way doors in the hierarchical equilibration model. Assymmetric molecular constraints impose that the ligand binds first to R1 and dissociates first from R2.

Figure 10. Evolutionary chain of molecular organization events starting from a state 0 (supposed to be a stable chemical soup) to the state $n$.

Figure 11. Examples of micro-reversible sigmoidal curves and their physiological roles. (a) Regulatable activity of aspartate transcarbamylase. (b) Oxygen transport by hemoglobin. 


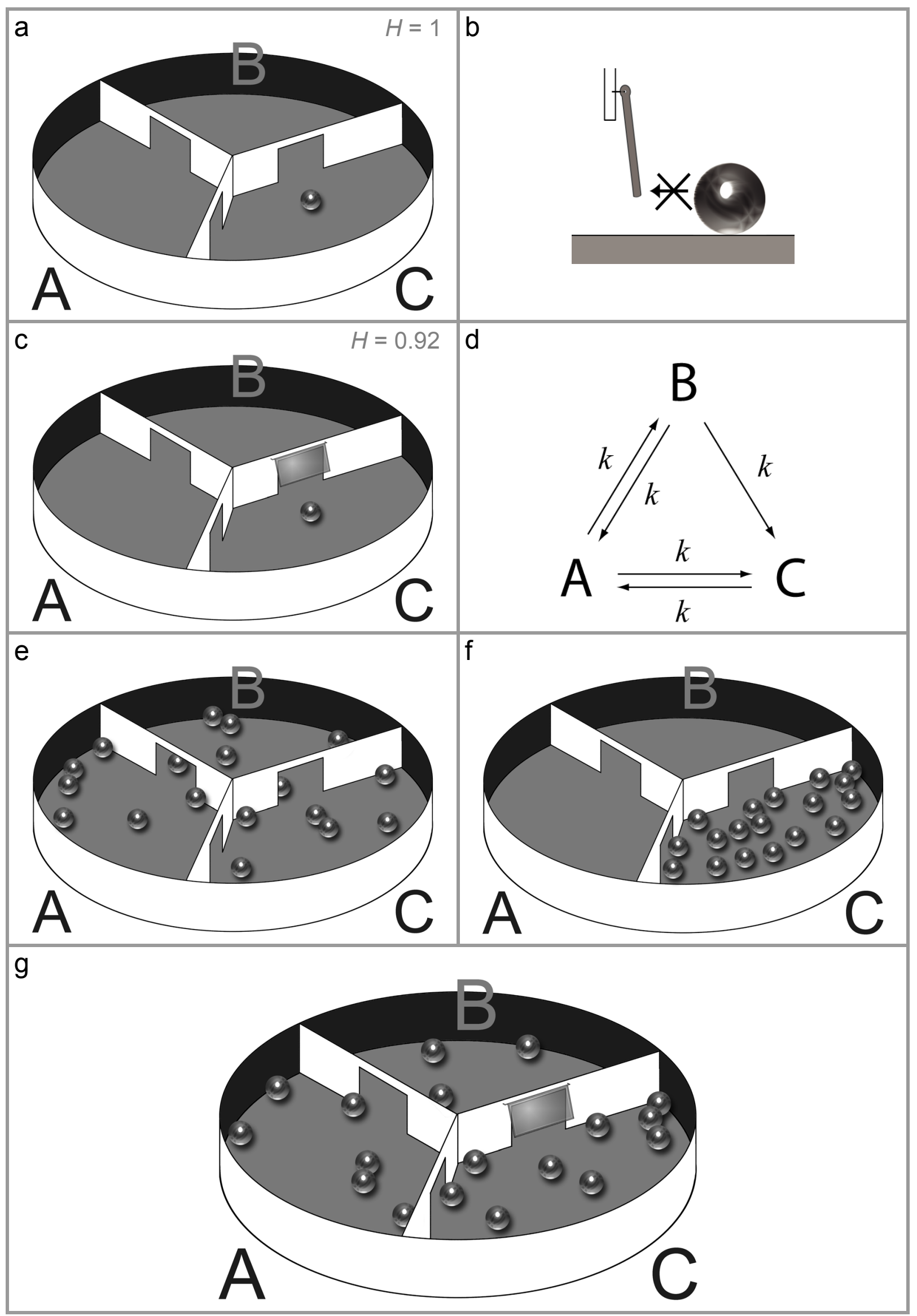

Figure 1 
a

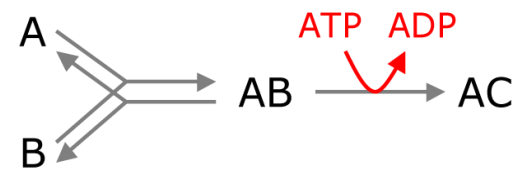

b

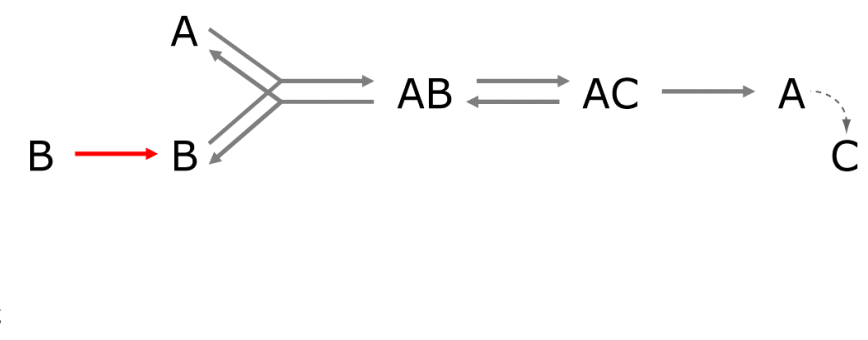

Gene $\stackrel{\bigcup}{\perp}$ Product $\longrightarrow \varnothing$

Figure 2 
a



b

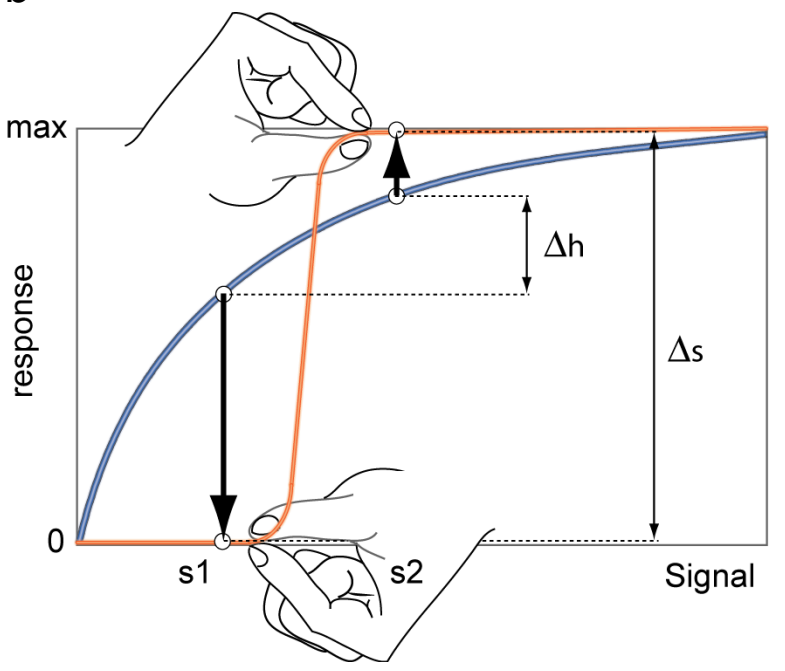

Figure 3 




Figure 4 
a

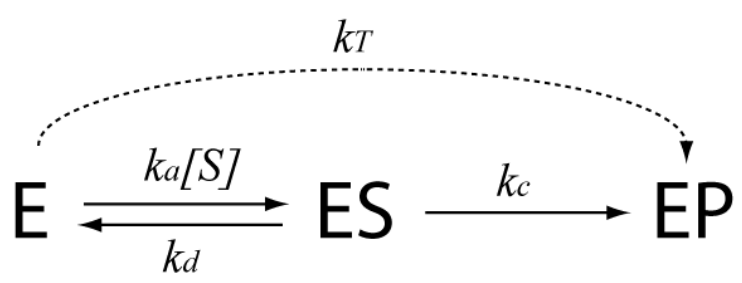

b

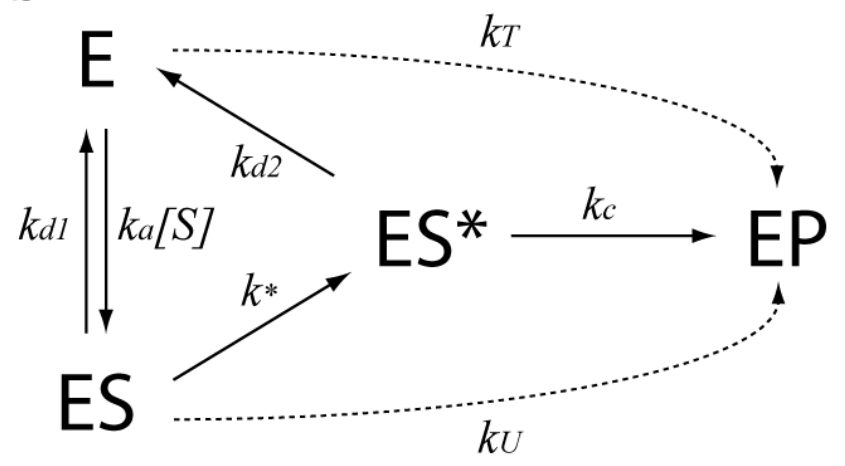

Figure 5 
a

$\mathrm{M} \underset{k d}{\stackrel{k a[L]}{\rightleftarrows}} \mathrm{ML}$<smiles>c1ccccc1</smiles>

b
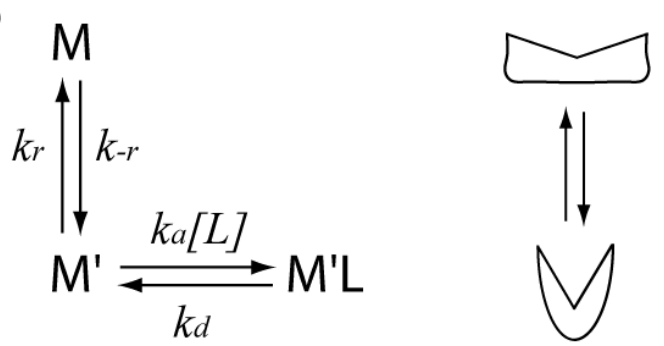

$Y \leftrightharpoons \nabla$

C
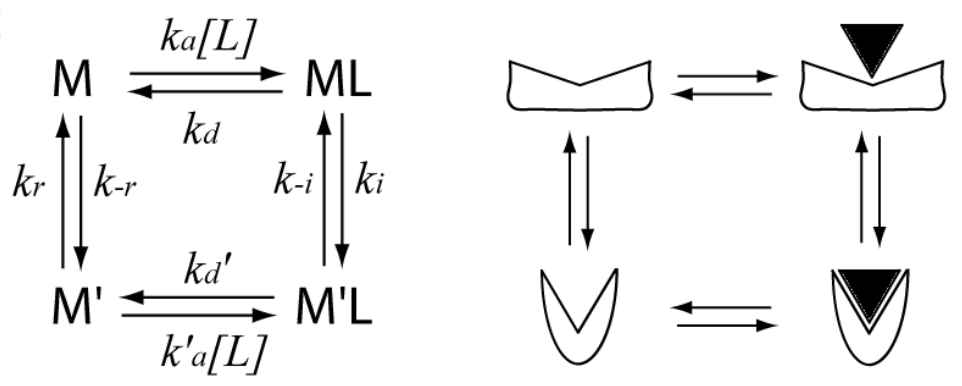

d

$$
\mathrm{M} \underset{k d}{\stackrel{k a[L]}{\rightleftarrows}} \underset{\mathrm{M}^{\prime} \mathrm{L}}{\stackrel{\mathrm{ML}}{\rightleftarrows}} \mathrm{ML}
$$

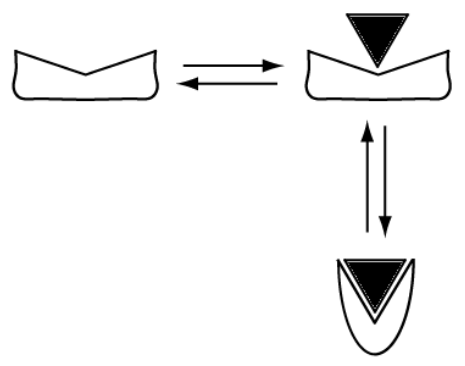

e

f

$$
\begin{aligned}
& \mathrm{E} \underset{k_{d}}{\stackrel{k_{a}[S]}{\rightleftarrows}} \mathrm{ES}
\end{aligned}
$$

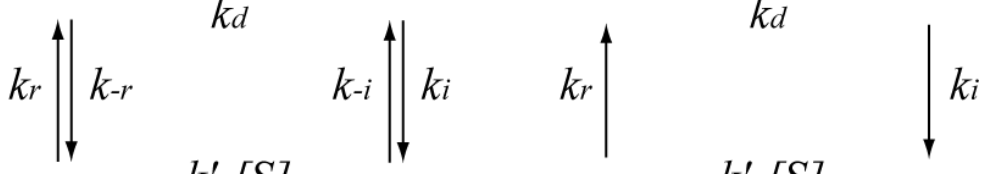

$$
\begin{aligned}
& \mathrm{E}^{\prime} \underset{k^{\prime} d}{\stackrel{k_{a}^{\prime}[S]}{\rightleftarrows}} \mathrm{E}^{\prime} \mathrm{\textrm {E } ^ { \prime }} \underset{k_{c}+k^{\prime} d}{\stackrel{k_{a}^{\prime}[S]}{\rightleftarrows}} \mathrm{E}^{\prime} \mathrm{S}
\end{aligned}
$$

Figure 6 
a

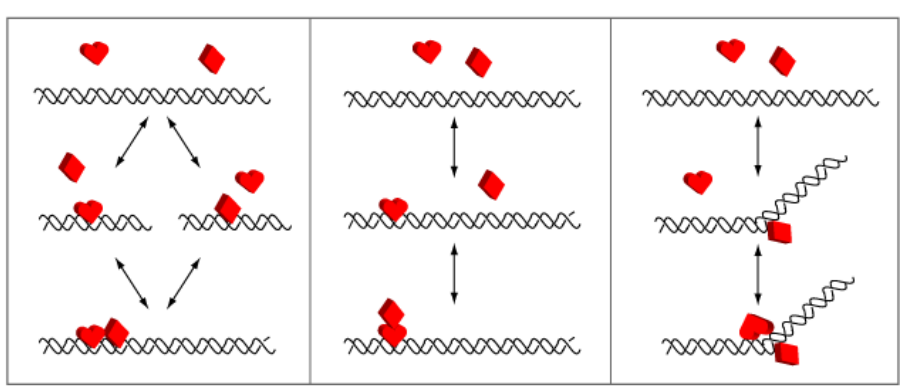

b
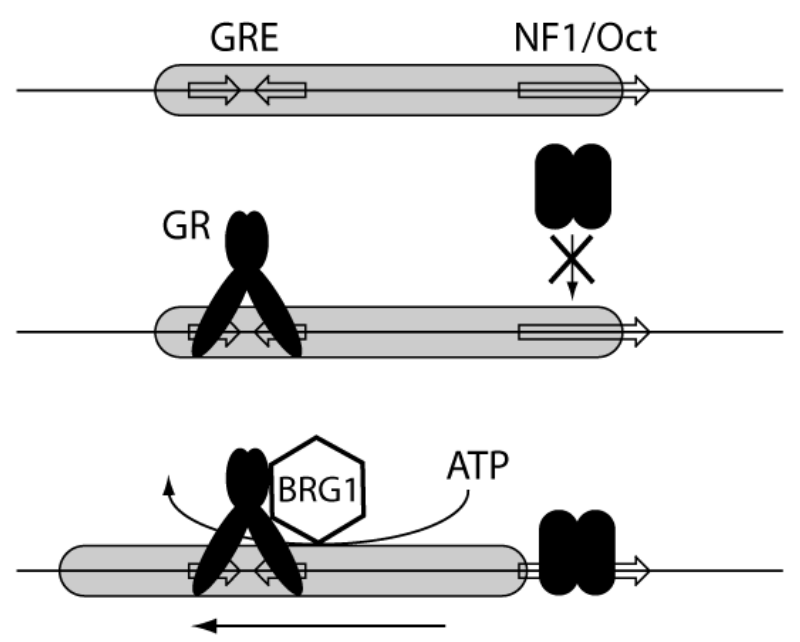

C

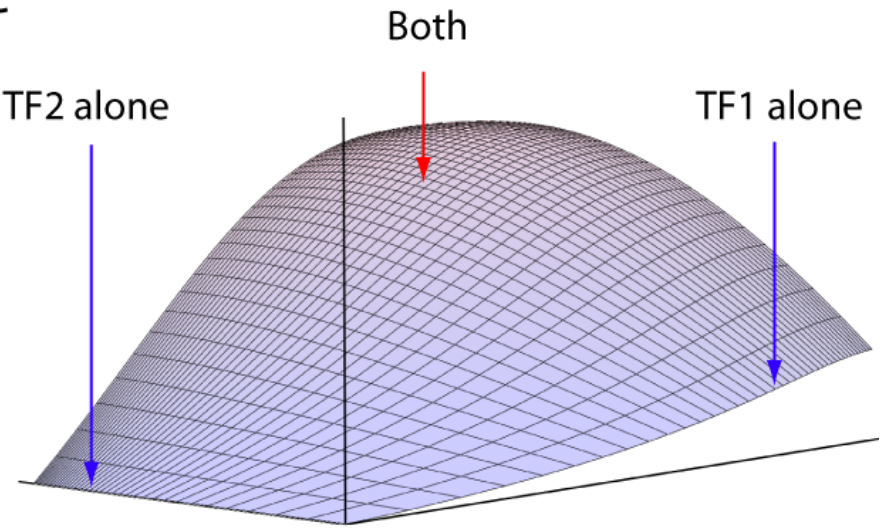

Figure 7 


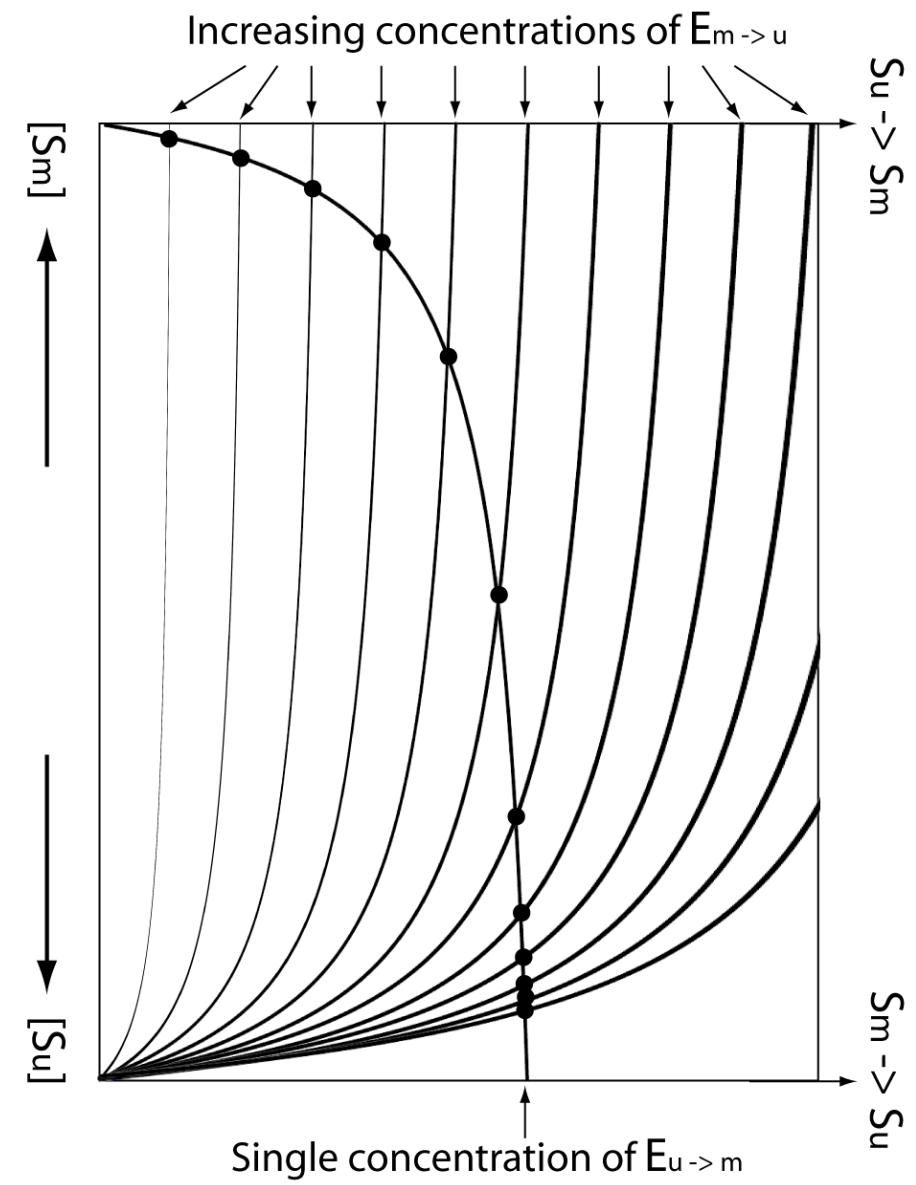

[Su]

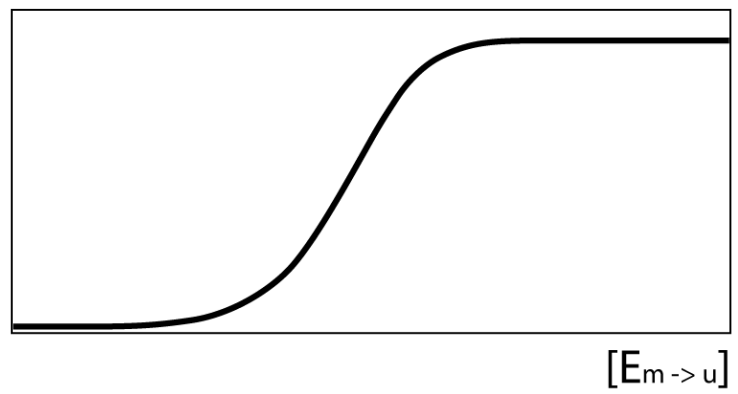

Figure 8 
a

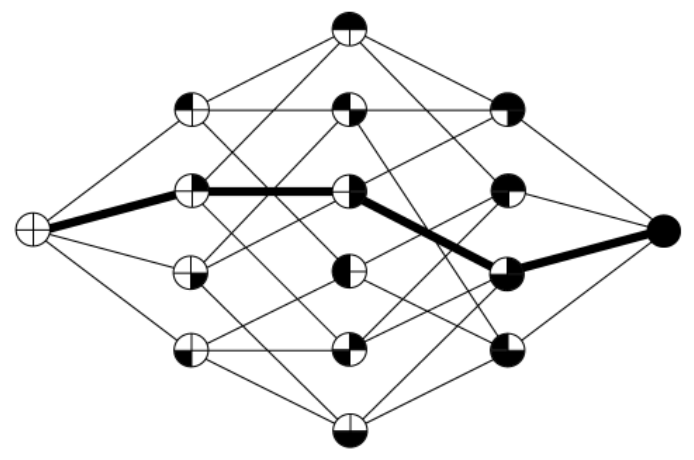

$\begin{array}{lllll}0 & 1 & 2 & 3 & 4\end{array}$

b

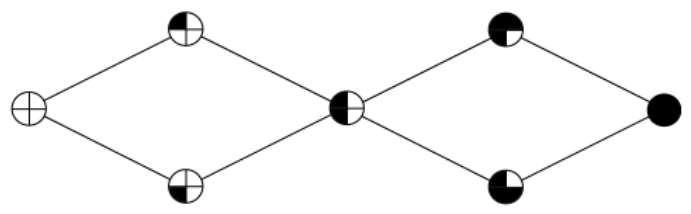

C

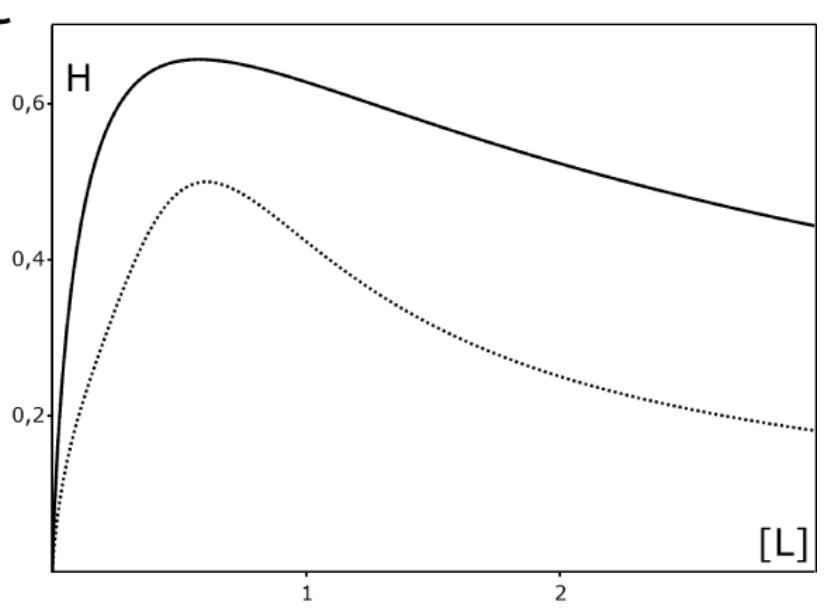

d

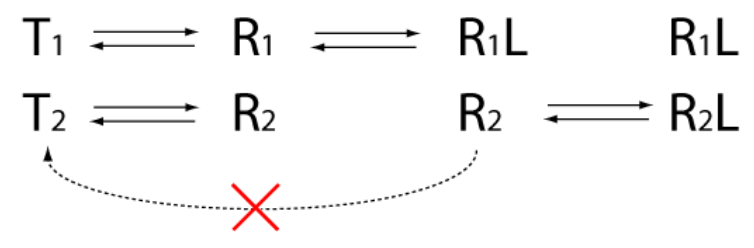

e

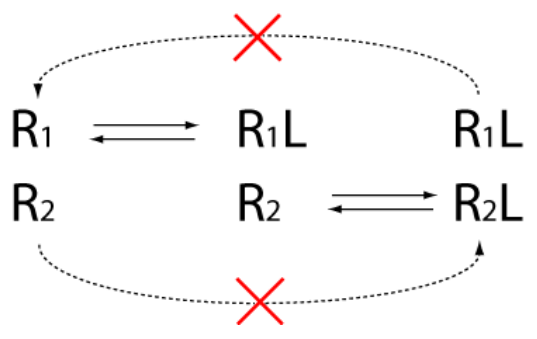

Figure 9 


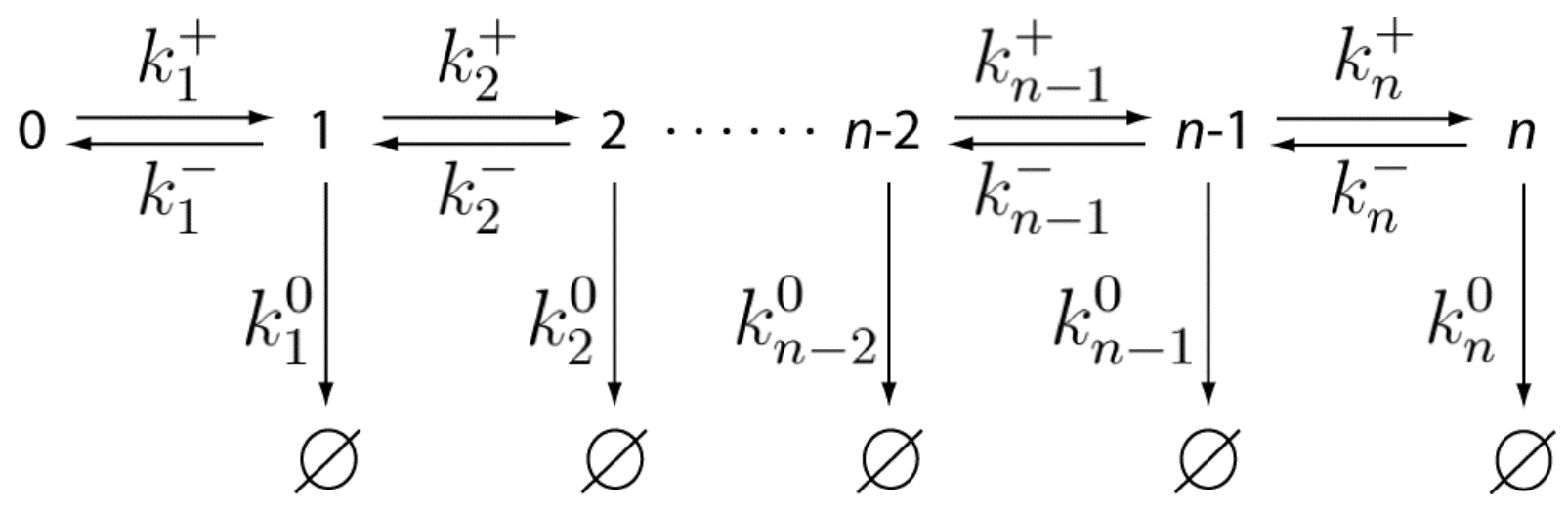

Figure 10 
a

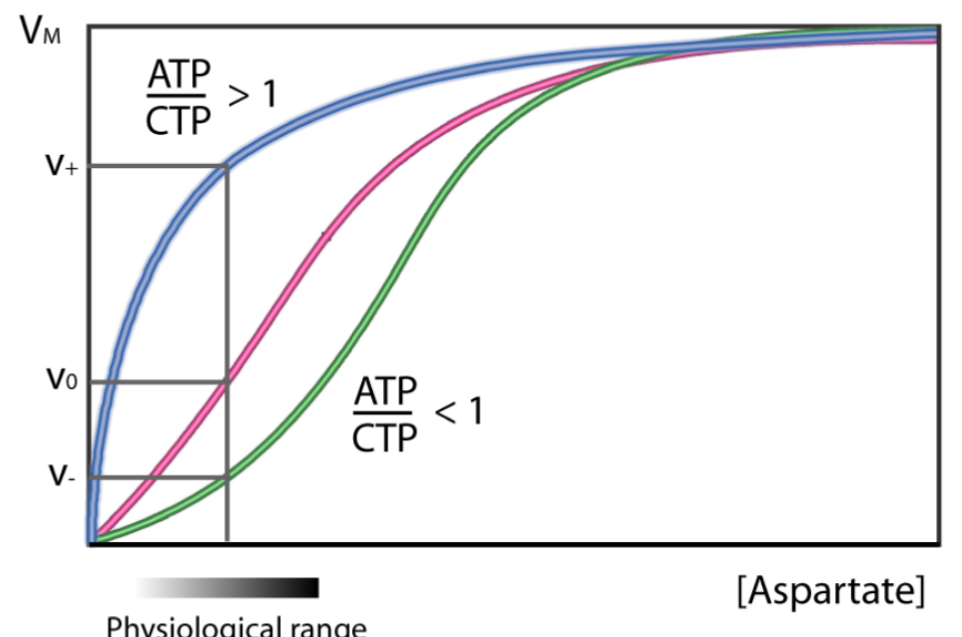

b

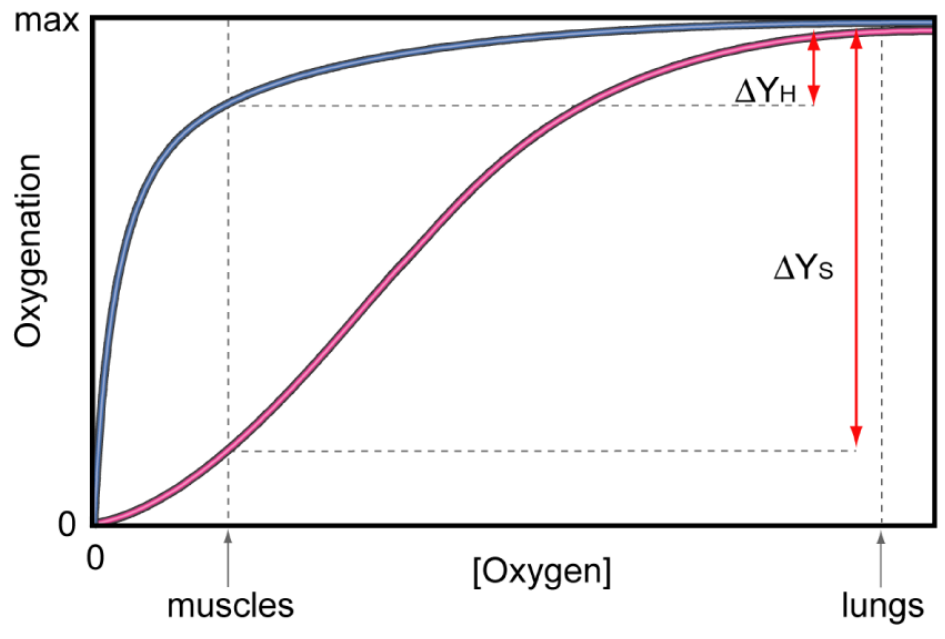

Figure 11 